\title{
Circulation and Exchange in Choked Marginal Seas
}

\author{
Larry J. Pratt and Michael A. Spall \\ Woods Hole Oceanographic Institution, Woods Hole, Massachusetts
}

(Manuscript received 25 October 2007, in final form 14 March 2008)

\begin{abstract}
A theory for the exchange between a rotating, buoyancy-forced marginal sea and an ocean is developed and tested numerically. Cooling over the marginal sea leads to sinking and sets up a two-layer exchange flow, with a warm surface layer entering from the ocean and a cool layer exiting at depth. The connecting strait is sufficiently narrow and shallow to cause the exchange flow to be hydraulically controlled. The incoming surface layer forms a baroclinically unstable boundary current that circles the marginal sea in a cyclonic sense and feeds heat to the interior by way of eddies. Consistent with the overall heat and volume balances for the marginal sea, there is a continuous family of hydraulically controlled states with critical flow at the most constricted section of the strait. Included in this family is a limiting "maximal-exchange" solution with two sections of hydraulic control in the strait and with fixed layer depths at the most constricted section. The state of exchange for a given forcing is predicted using a theory that assumes energy conservation over a certain path connecting the strait to the marginal sea or, in some cases, the ocean. Depending on the configuration of the exchange, long-wave information may be blocked from entering the strait from the marginal sea, from the open ocean, or both. The scenario that holds determines what is predicted and what needs to be input. Numerical tests of the prediction for the temperature difference and the state of exchange are carried out for straits with a pure contraction in width and for a constant width strait with a topographic sill. The comparison is reasonable in most cases, though the numerical model is not able to reproduce cases of multiple states predicted by the theory for certain forcing values. The analytical model is an alternative to the Price and Yang and Siddall et al. models of a marginal sea outflow.
\end{abstract}

\section{Introduction}

The buoyancy-forced circulation in a marginal sea and the corresponding mass and heat exchange with the open ocean have been the subject of recent attention (e.g., Price and Yang 1998; Siddall et al. 2002, 2004; Spall 2003, 2004; Walin et al. 2004; Straneo 2006). In a set of numerical experiments particularly relevant to the present work (Spall 2004), the marginal sea is subjected to a surface heat loss, producing a cool interior water mass. The heat is supplied by a warm inflow that enters from the open ocean through the strait and forms a boundary current that circles the basin cyclonically. The boundary current is baroclinically unstable and produces eddies that carry heat into the interior, where it is lost through the surface. The resulting

Corresponding author address: Larry Pratt, Woods Hole Oceanographic Institution, MS\# 21, 360 Woods Hole Rd., Woods Hole, MA 02543.

E-mail: lpratt@whoi.edu cooler, denser water mass drains out of the marginal sea and into the ocean, setting up an exchange flow in the connecting passage. This passage is typically much wider than the Rossby radius of deformation, and thus the exchange flow is not constricted or choked in any way. The general situation is applicable to semienclosed bodies of water like the Labrador Sea (Straneo 2006), which has extensive lateral boundaries but unrestricted access to the open ocean. An extension of this approach to marginal seas separated from the open ocean by a sill for which choking does not occur is carried out by Iovino et al. (2008).

There are many examples in which the strait or gap connecting the inland basin to the ocean is narrow and the exchange is restricted. Choking of the flow can result from hydraulic or frictional effects; we will concentrate on the former. Examples range from estuaries, usually too small to be influenced by rotation, to large inverse estuaries such as the Mediterranean and Red Seas. Here, the connecting straits are sufficiently narrow that rotation can be neglected within, but the basins themselves are generally wide enough to permit 
geostrophic boundary currents. The purpose of this work is to establish a simple theory for these largerscale applications, one that can predict the state of exchange and the corresponding volume fluxes given the thermal forcing over the basin and the outside ocean surface temperature. The theory will combine ideas about generic marginal sea circulations, largely motivated by past numerical simulations and theory (Spall 2004), with two-layer hydraulic theory in the strait (e.g., Armi and Farmer 1986) and some ideas about estuary circulation that date back to Stommel and Farmer (1953). Our two-layer theory will be compared with numerical simulations of a continuously stratified, thermally forced marginal sea, connected to the ocean by a strait sufficiently narrow that rotation can be ignored within.

The models presented herein are highly idealized and not, at this stage, meant to simulate the Mediterranean Sea, Red Sea, or any other marginal sea. We do not, for example, attempt to reproduce the complicated geometric and topographic details of these basins and straits. In addition, we restrict attention to thermal forcing and neglect the evaporation effects that are often important in applications. The outflow temperatures predicted will appear unrealistic, though not necessarily so when converted into density differences. All of these simplifications are made to allow for the development of an analytic model that is tractable and not weighed down by lengthy algebra. The major ingredients (twolayer hydraulic control, geostrophic boundary layers, etc.) are thought to be robust and universal.

The major results are described in sections 2 and 3. The first lays out the theory, while the second describes comparison with numerical simulations. The theory section begins (section 2a) with a review of two-layer hydraulics with no rotation. It describes how the possible solutions with hydraulic control differ depending on whether the choking is due to a contraction in the width of the strait or to a shallow sill. In either case, there exists a family of solutions that has different volume exchange rates and layer thicknesses in the strait. Each family contains a limiting maximal solution that has the greatest amount of volume exchange that can be forced through the strait for a particular value of the reduced gravity (or layer temperature difference). To relate these exchange flows to the marginal sea state, one must use various constraints based on volume, heat, and mechanical energy budgets for the marginal sea. These are described in section $2 b$. The budgets for heat and mass can be used to define a family of coupled states (section 2c) in which a hydraulically controlled exchange is linked to a marginal sea forcing and to the temperature difference between the two layers. Stom- mel and Farmer (1953) first derived this relationship (in the slightly different context of a salinity-stratified estuary flow) and suggested that the state corresponding to maximal exchange would be achieved by increasing the forcing (in their case, wind and tidal mixing). If the forcing is increased beyond the point where maximal exchange sets in, the basin is said to be overmixed. Although the idea of overmixing is less relevant in our thermally forced model, the concept of maximal exchange is quite important.

At this stage of development, the problem is not yet closed. To predict the exchange state that occurs for a particular forcing and to forecast the forcing required to drive the system to maximal exchange, it is necessary to use a further constraint along with some assumptions about the nature of circulation in the marginal sea. Although this has been attempted in nonrotating systems (e.g., Brocard and Harleman 1980; Finnigan and Ivey 1999, 2000; Whitehead et al. 2003; Grimm and Maxworthy 1999), the only previous attempts to do so in a rotating system are apparently those of Siddall et al. (2002, 2004, and references therein) and Price and Yang (1998). The latter consider a marginal sea with a deep, quiescent lower layer that spills out and over a shallow sill. The sill flow feels rotation and has zero potential vorticity. The layer above is assumed to be sluggish and inactive, and the exchange is always submaximal. Closure is made through specification of the depth of the interface in the marginal sea, which is assumed to be constant. Our marginal sea has an active circulation with boundary currents. The connecting strait is sufficiently narrow to minimize rotational effects, but both layers are active there, which allows for maximal and submaximal exchange states. Closure results from the enforcement of energy conservation along a short segment of coastline extending from the most constricted section of the strait into the marginal sea. The result is that one need only to specify the buoyancy forcing in the marginal sea (and not interface depth).

In the Siddall et al. $(2002,2004)$ model, hydraulic control is made possible by a shallow sill that lies between the marginal sea and the open ocean, and the corresponding submaximal states block information from propagating from the ocean into the marginal sea. In addition to states subject to control by a shallow sill, we consider exchange flows that are controlled by sills of different depths as well as states controlled by a pure sidewall contraction. The latter brings into play a new family of submaximal states for which the direction of information propagation is reversed. To close their calculation, Siddall et al. $(2002,2004)$ utilize a parameterization of buoyancy flux between the strait and the in- 
terior, leading to dependence on an eddy diffusivity coefficient. Our energy approach (section 2d) avoids this dependence but uses a conceptual model for the geostrophic boundary current that forms in the marginal sea. The result is independent of eddy coefficients, although it does depend on an $O(1)$ coefficient that relates the boundary current width to the Rossby radius of deformation. The assumption of conservation of energy may fail for exchange states that contain a hydraulic jump at the marginal sea end of the strait. (The corresponding submaximal states permit information propagation into, but not out of, the marginal sea.) In such cases, the theory can be adjusted to conserve energy between the most constricted section and the open ocean (section 2e). Implementation, therefore, depends on the direction of information propagation through the strait, which must be known in advance.

Our theory also differs in several respects from the analytical model of noncontrolled exchange in Spall (2004). First, the presence of hydraulic control provides an extra constraint, namely, a critical condition. One consequence is that the thickness of the inflowing layer in the strait is now predicted rather than assumed. The extra constraint is nonlinear, which leads to other novel aspects including the possibility of multiple states for fixed forcing and the abovementioned dependence on the direction of information propagation into or out of the marginal sea. When the strait is wide and no hydraulic effects occur, information is free to pass through in either direction and there is no such dependence.

The numerical simulations (section 3) show good qualitative agreement with the theory in most cases. The experiments are carried out using two basic strait geometries, one with a topographic sill and constant width and the other with a flat bottom and a smooth width contraction. As discussed in the next section, the permissible two-layer hydraulic solutions for the two cases can be quite different. The key dimensionless parameter in the analytic model depends, among other things, on the forcing and strait width. This parameter is adjusted in an attempt to drive conditions toward the maximal exchange limit. This limit is apparently achieved when the exchange flow is controlled by a sill. The approach to maximal exchange in this case is through a series of submaximal states in which information propagation into the marginal sea from the open ocean is blocked. However, when we attempt to make the approach through a sequence of submaximal states in which the flow of information is reversed, possible for cases of a pure contraction, maximal exchange is apparently achieved somewhat before the theoretical threshold. We mention some possible explanations.

\section{Theory}

The theory for the marginal sea and strait system combines two-layer hydraulic theory in the strait with thermodynamic and mass balances for the marginal sea as a whole. The theory also depends on some general assumptions about the horizontal circulation in the marginal sea (Fig. 1). The overall picture is suggested by present and past numerical simulations (e.g., Spall 2004) but, more importantly, is consistent with elementary geophysical fluid dynamics principles. The key assumption is that the inflowing upper layer, after entering the marginal sea through the strait, hugs its righthand (Northern Hemisphere) coast and forms a boundary current that travels cyclonically around the basin edge. In Spall (2004), this current is baroclinically unstable and loses heat to the interior of the marginal sea through eddy fluxes. If the basin is sufficiently large, the current will lose all its excess heat to eddy fluxes and will entirely dissipate before it has circled the basin (Fig. 1a). Although we will focus on this scenario, the small basin case of a boundary current that circumnavigates the basin edge and sets up a recirculating rim current (Fig. 1b) can also be handled (appendix A), but the resulting theory depends on an eddy heat flux coefficient.

\section{a. Review of two-layer exchange flows}

Before laying out the theory for the marginal sea, it will be helpful to review some of the basic properties of two-layer exchange flow in a nonrotating strait. The constraints to be used are based on the steady shallowwater equations for a Boussinesq two-layer fluid under a rigid lid:

$$
\begin{aligned}
v_{1} \frac{\partial v_{1}}{\partial y} & =-\frac{1}{\bar{\rho}} \frac{\partial p_{T}}{\partial y}, \\
v_{2} \frac{\partial v_{2}}{\partial y} & =-\frac{1}{\bar{\rho}} \frac{\partial p_{T}}{\partial y}-g^{\prime}\left(\frac{\partial d_{2}}{\partial y}+\frac{\partial h_{b}}{\partial y}\right), \\
\frac{\partial\left(w v_{n} d_{n}\right)}{\partial y} & =0 .
\end{aligned}
$$

Here, $v_{n}, d_{n}$, and $\rho_{n}$ are the velocities, depths, and densities of layers 1 (top) and 2 (bottom), $h_{b}$ is the bottom elevation, $p_{T}$ is the rigid lid pressure, $g^{\prime}=g\left(\rho_{2}-\rho_{1}\right) / \rho_{\mathrm{o}}$, $\rho_{\mathrm{o}}$ is a representative density, $w$ is the channel width, and $y$ increases in the direction of the marginal sea. [For reviews of the development of the central ideas and constraints of two-layer hydraulics, the reader might consult Baines (1995) or Pratt and Whitehead (2008).]

The strait separating the marginal sea from the ocean will take on one of two basic configurations. In the first 


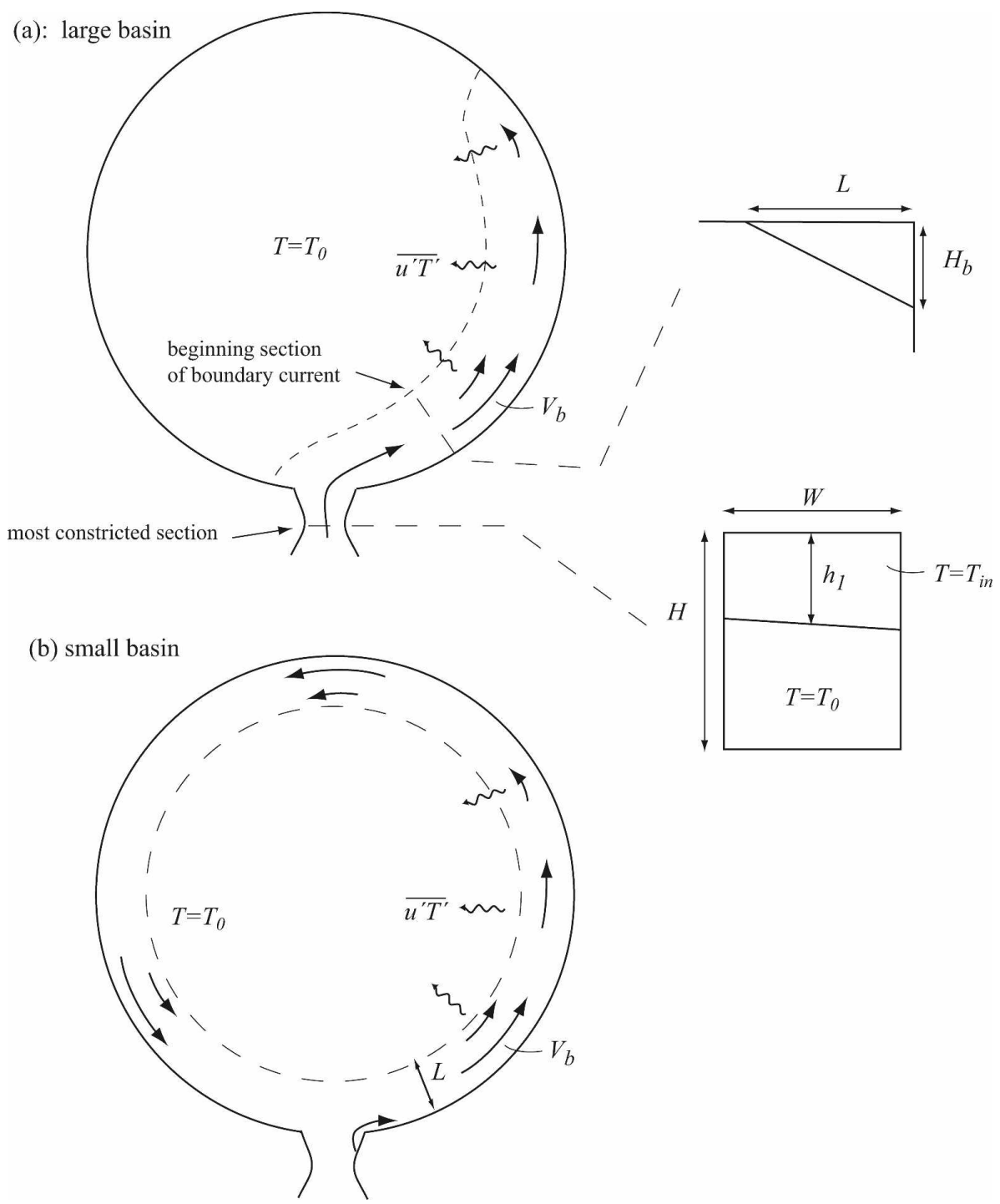

FIG. 1. Definition sketch. (a) The marginal sea with a boundary current that terminates before circling the basin. The lower inset sketch shows the expected cross section of the two-layer system at the most constricted section. The width $W$ there is sufficiently narrow such that rotation is weak. The upper-inset sketch shows the cross section of the boundary current at a section where it has just formed. (b) The marginal sea with a boundary current that circles the basin completely.

(Fig. 2a), the bottom depth remains constant, but the width smoothly decreases to a minimum value $w=W$ at the narrowest section. In the second case (Fig. 3), the width remains fixed, but the bottom smoothly shoals to a minimum water depth $H$. In either case, the strait is sufficiently constricted such that the exchange flow is "choked," or hydraulically controlled. In addition, the width of the strait at its most constricted section is assumed to be small in comparison to the internal Rossby radius of deformation. Tilting of the interface across the strait (Fig. 1a, inset) and other possible effects of rotation are therefore considered sufficiently weak to be neglected. The layer depths and velocities at this section, designated $h_{n}$ and $V_{n}$, are therefore considered uniform across the strait. This uniformity is consistent with the use of the above one-dimensional shallow water equations.

Hydraulic control in the presence of smooth variations in width or depth occurs when the flow becomes critical; that is, when a long, internal gravity wave that would otherwise propagate through the section in question becomes arrested. This condition occurs when the composite Froude number becomes unity,

$$
G^{2}=\frac{V_{1}^{2}}{g^{\prime} h_{1}}+\frac{V_{2}^{2}}{g^{\prime}\left(H-h_{1}\right)}=1,
$$



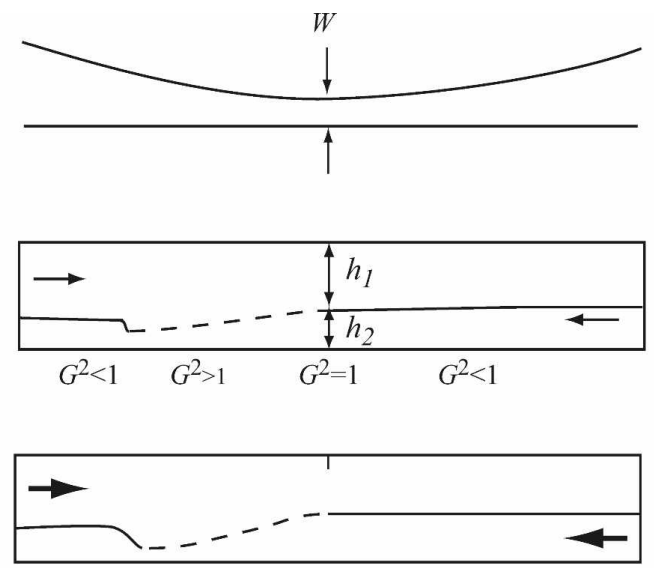

$$
G^{2}<1 \quad G^{2}>1 \quad G^{2}=1 \quad G^{2}<1
$$
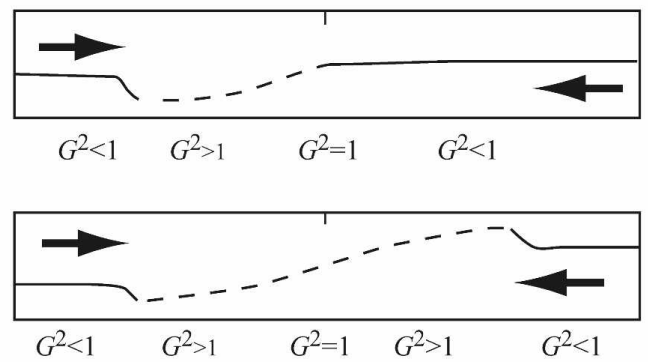

(f):

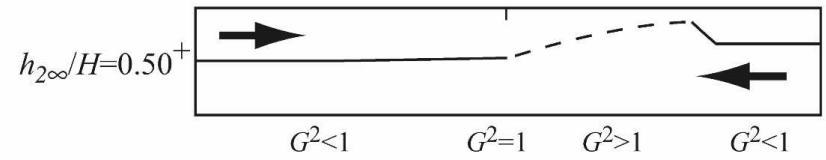

(g): $h_{2 \infty} / H=0.52$

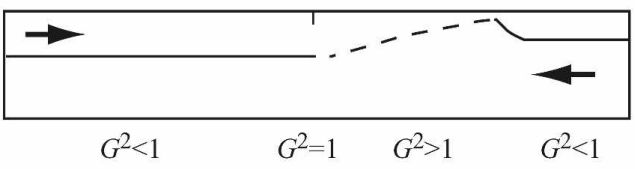

(h)

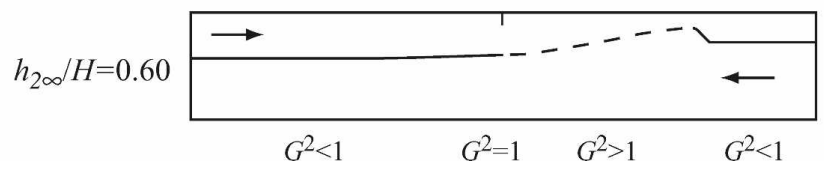

FIG. 2. A selection of hydraulically controlled two-layer exchange flows in a channel with a horizontal bottom and a width contraction, as shown in the top. (e) The case of maximal exchange. Here $G$ is the composite Froude number, as defined by (1). (Based on Fig. 2 in Armi and Farmer 1986.)

and is often restricted to the most constricted section, here the narrowest or shallowest. In special cases to be described below, a second section of critical flow exists.

Under conditions of pure exchange flow (no net volume transport) in a channel with a constant depth and a smooth width contraction, there is a family of solutions that become critical in the narrowest section (Figs. $2 \mathrm{~b}-\mathrm{h})$. It is convenient to imagine that these flows are connected to infinitely wide and stagnant reservoirs at each end. Consider the case in which the lower layer is fed from the right-hand reservoir, where the lowerlayer depth is $h_{2 \infty}$. When the reservoir interface lies below middepth $\left(h_{2 \infty} / H<0.5\right)$, the lower layer accelerates and shallows slightly as it enters the strait (Fig. 2b). A subcritical-to-supercritical transition occurs as the narrowest section is passed and $G^{2}$ increases from below to above unity. The supercritical flow that occurs to the left of the narrowest section (indicated by a dashed interface) often terminates in an internal hydraulic jump. Long internal waves may propagate 


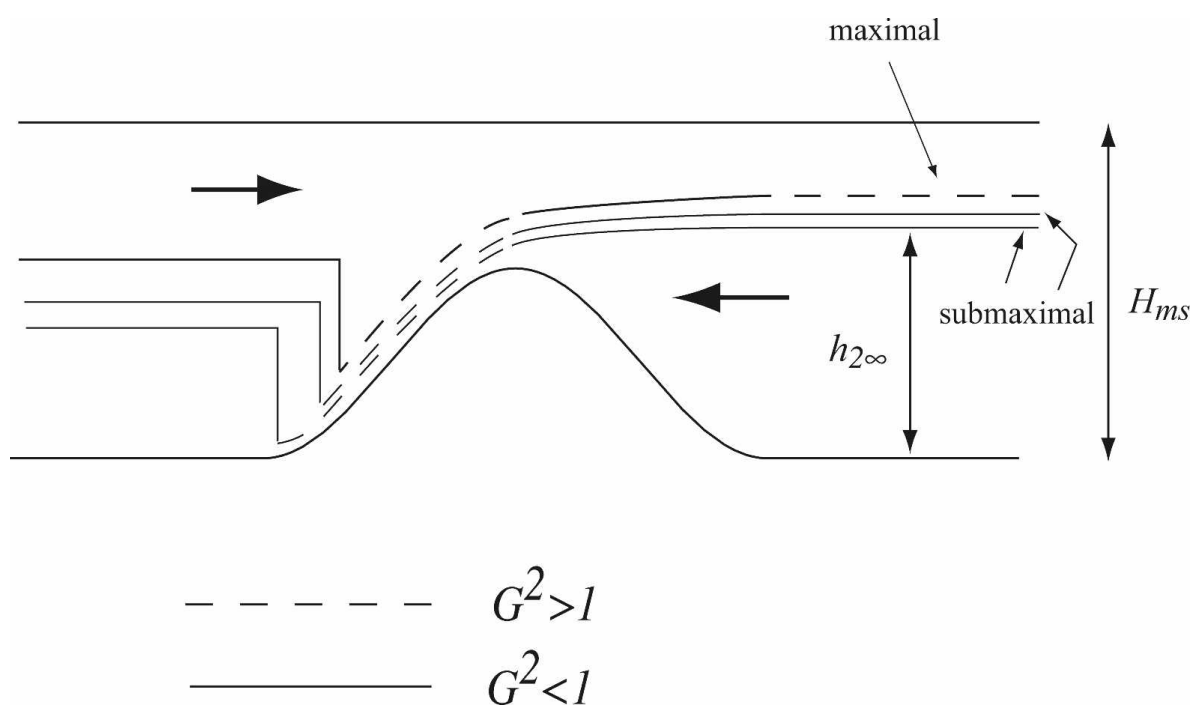

FIG. 3. Maximal and submaximal two-layer states for flow over an obstacle in a channel with constant width.

through the strait from right to left, but waves entering the strait from the left are blocked by the supercritical flow. For each solution with $h_{2 \infty} / H<0.5$ (Fig. 2b-d), there are similar solutions (Fig. $2 \mathrm{f}-\mathrm{h}$ ) in which the behavior of the layers is reversed. In such cases, the direction of information flow in the form of waves is reversed and $h_{2 \alpha} / H$ is measured in the left-hand reservoir. For example, the solution for $h_{2 \infty} / H=0.4$ has a symmetrical counterpart $h_{2 \infty} / H=0.6$ in which the upper layer is relatively thin and goes through the same transitions as the lower layer in the previous case.

These tendencies remain and the volume exchange rate increases as the controlling reservoir interface tends toward the middepth level $h_{2 \infty} / H=0.5$. However, this limit leads to a solution (Fig. 2e) with qualitatively new properties. The new flow is what would take place as the result of a classical "lock exchange" experiment, in which the right and left reservoirs are filled with fluid of different densities and a barrier separating the two bodies is removed. The lock exchange solution is critical at the narrowest section but supercritical immediately to the left and right. Internal wave propagation is outward, away from the critical section, so that information from either reservoir is prevented from influencing the flow in the narrows or in the opposite reservoir. The lock exchange solution has the largest volume exchange rate of all the solutions and is referred to as having maximal exchange. Cases with $h_{2 \infty} / H \neq 0.5$ are referred to as having submaximal exchange.

When the strait contains an obstacle and has uniform width, the exchange flows are quite different (Fig. 3). Variations in bottom topography are felt directly only in the lower layer, and the symmetry properties of the previous solutions are lost. In particular, the group of solutions having a relatively shallow upper layer is no longer present. One may again assume that the lower layer is fed from an infinitely wide reservoir to the right. For small $h_{2 \infty}$ the lower layer spills over the sill, becomes supercritical, and undergoes a hydraulic jump, all in the manner of a single-layer flow over a dam. As $h_{2 \infty}$ increases, the lower layer thickens, the volume exchange increases, and the upper layer becomes thinner. At a limiting value of $h_{2 \infty}$, which depends on the ratio of the sill depth $H$ to the upstream channel depth $H_{\mathrm{ms}}$, the flow to the right of the obstacle becomes critical. The exchange flow has now reached its maximal state and no solution exists for larger $h_{2 \infty}$. In practice, the channel often broadens into a wider body of water somewhere to the right of the obstacle. In this case, the uniform stretch of critical flow that is predicted to the right of the obstacle becomes supercritical, which is implied in Fig. 3. As was the case for a pure contraction, the two stretches of supercritical flow cause internal gravity waves to propagate outward, away from the strait and into the reservoirs. Also, there are now two distinct sections of critical flow, one at the sill of the obstacle and the other just to the right of the obstacle.

A thorough discussion of the above solutions can be found in Armi (1986) and Armi and Farmer (1986). When channel width variations occur in combination with topographic variations, the situation becomes more complicated (Farmer and Armi 1986; Dalziel 1991). However, it is always possible to find a family of submaximal solutions with a single critical section and a limiting maximal solution with two critical sections. (For the above case of maximal pure exchange through 
a pure contraction, the two critical sections coincide.) The submaximal solutions permit the flow of information via long internal waves through the strait in one direction only, whereas the maximal solution permits no information to propagate through.

\section{b. Connecting the strait and marginal sea}

The above hydraulic solutions, valid in the strait, can be connected to the circulation in the basin using budgets for volume, heat, and energy. In this subsection we will simply write the corresponding expressions down along with a few other useful constraints. Forcing of the system is entirely thermal, and the effects of evaporation in the marginal sea (along with the implied net volume inflow) will be ignored. Let $Q$ represent the uniform surface heat flux per unit area (positive for heat loss to atmosphere) over the marginal sea. Although the numerical simulations allow for more continuous vertical variations in density and velocity, the analytical model will treat the exchange flow in the strait and certain elements of the marginal sea circulation within a two-layer formalism. The heat balance for the marginal sea as a whole then becomes

$$
\begin{aligned}
\frac{A Q}{\rho_{o} c_{p}} & =V_{1} h_{1} W T_{1}+V_{2}\left(H-h_{1}\right) W T_{2}, \\
& =V_{1} h_{1} W\left(T_{1}-T_{2}\right),
\end{aligned}
$$

where $A$ is the surface area of the marginal sea and $T_{1}$ and $T_{2}$ are temperatures of the inflowing and outflowing layers, respectively, taken at the most constricted section in the strait. The statement of zero net volume inflow,

$$
V_{1} h_{1} W+V_{2}\left(H-h_{1}\right) W=0,
$$

has been used in the second step.

Consider the boundary current at a location within several deformation radii of the entrance to the strait, where the mean upper layer velocity scale is $V_{\mathrm{b}}$ and the width is $L$ (Fig. 1a, inset). It is assumed that the flow here is in geostrophic balance and that the lower layer is relatively quiescent. ${ }^{1}$ The thermal wind balance for the current is then

\footnotetext{
${ }^{1}$ This assumption can be motivated by inspection of the hydraulic solutions in Figs. $2 \mathrm{f}-\mathrm{h}, 3$, all of which have relatively deep lower layers and small lower-layer velocities at the right-hand exit of the strait. The lower layers are relatively deep and inactive at this location, a feature that supports the neglect of lower-layer motion (or grounding of the interface at the wall) just inside the marginal sea. The neglect of the lower layer is more questionable for maximal solutions with hydraulic jumps or the solutions shown in Figs. 2b-e. A special theory for the latter is presented later in the paper.
}

$$
V_{b}=\frac{\alpha g H_{b}}{\rho_{o} f L}\left(T_{1}-T_{2}\right),
$$

where $H_{b}$ is the upper-layer depth at the wall and $\alpha$ is the coefficient of thermal expansion for seawater. Following Spall (2004), $L$ is assumed to be proportional to the Rossby radius of deformation, in particular,

$$
L^{2}=\kappa^{2} g^{\prime} H_{b} / f^{2}=\kappa^{2} \alpha g\left(T_{1}-T_{2}\right) H_{b} / \rho_{o} f^{2},
$$

where $\kappa$ is an $O(1)$ constant. The second step uses a linear equation of state to express reduced gravity in terms of temperature difference:

$$
g^{\prime}=g \alpha \frac{\left(T_{1}-T_{2}\right)}{\rho_{o}},
$$

where $\alpha$ is the coefficient of thermal expansion.

The properties $V_{b}, L$, etc., are assumed to be measured sufficiently close to the strait such that properties like volume flux and energy are conserved between this location and the most constricted section of the strait. Conservation of volume flux for the upper layer implies that

$$
Q_{v}=V_{1} h_{1} W=g^{\prime} H_{b}^{2} / 2 f,
$$

where again $V_{1}, h_{1}$, and $W$ are measured at the most constricted section. The final term in (7) is just the volume flux of a geostrophic boundary current with layer thickness $H_{b}$ at the wall and with zero layer thickness offshore (Fig. 1, inset). The Bernoulli function within each layer is also assumed to be conserved between the strait and the upstream portion of the marginal sea boundary layer. We will specifically consider the internal energy, the difference between the upperand lower-layer Bernoulli functions. Because the Bernoulli function for a particular layer is conserved along streamlines within that layer, the internal energy is conserved along a wall or any other path over which upperand lower-layer streamlines coincide. Equating the internal energy at the most constricted section of the strait to the wall value of internal energy for the boundary layer leads to

$$
\Delta B \equiv \frac{V_{1}^{2}}{2}-\frac{V_{2}^{2}}{2}+g^{\prime} h_{1}=\frac{V_{b}^{2}}{2}+g^{\prime} H_{b} .
$$

\section{c. Maximal and submaximal states}

It will now be shown that the possible coupled states of circulation in the basin and strait system consist of a family of solutions with varying volume exchange rates, including a limiting maximal exchange solution. Following the Stommel and Farmer (1953) ideas for an estuary, we first write down a Kundsen-like relation for temperature based on (2) and the first equality in (7): 


$$
Q_{v}\left(T_{1}-T_{2}\right)=\frac{A Q}{\rho_{o} c_{p}} .
$$

In addition, it is possible to use (3) to rewrite the critical condition (1) in the form

$$
\frac{Q_{v}^{2}}{g^{\prime} h_{1}^{3} W^{2}}+\frac{Q_{v}^{2}}{g^{\prime}\left(H-h_{1}\right)^{3} W^{2}}=\frac{Q_{v}^{2}}{g^{\prime} W^{2}}\left[\frac{1}{h_{1}^{3}}+\frac{1}{\left(H-h_{1}\right)^{3}}\right]=1 .
$$

Elimination of $Q_{v}$ between the last two equations and use of the expression (6) for $g^{\prime}$ leads to

$$
\Delta \tilde{T}^{3}=\frac{1}{\left(h_{1} / H\right)^{3}}+\frac{1}{\left(1-h_{1} / H\right)^{3}},
$$

where

$$
\Delta \tilde{T}^{3}=\frac{\alpha g W^{2} \rho_{o} c_{p}^{2} H^{3}\left(T_{1}-T_{2}\right)^{3}}{A^{2} Q^{2}}
$$

is a nondimensional temperature difference between layers. The temperature scale

$$
\left(\frac{A^{2} Q^{2}}{\alpha g W^{2} \rho_{o} c_{p}^{2} H^{3}}\right)^{1 / 3}
$$

can be shown to be the temperature difference that would occur if the inflow and outflow each had depth $H$ and speed $V_{\text {in }}$ equal to $\left(g^{\prime} H\right)^{1 / 2}$. In reality, the actual depth of each layer must be $<H$, and $V_{\text {in }}$ must be $<\left(g^{\prime} H\right)^{1 / 2}$, so $\left(T_{1}-T_{2}\right)$ will always exceed the temperature scale.

Equation (10) is similar to what Stommel and Farmer (1953) and later authors (e.g., Bryden and Stommel 1984) have obtained in connection with models of estuaries and inverse estuaries. There the forcing is usually treated as haline rather than thermal, but this is felt only in the nondimensionalization. [If river runoff or evaporation becomes a significant contributor to the volume flux budget, the equation itself becomes a little more complicated.] For the present model, a plot of $\Delta \tilde{T}$ over the full depth range $0<h_{1} / H<1$ gives a U-shape curve (hereafter referred to as the U curve) with a minimum value $\Delta \tilde{T}=2\left(2^{1 / 3}\right)$, as shown in Fig. 4 . A flow state lying on the curve to the right of the minimum has a relatively deep surface layer $\left(h_{1} / H>0.5\right)$ at the most constricted section of the strait, whereas the opposite is true for states on the left-hand branch. At $h_{1} / H=0.5$, the layer depths are equal and $\Delta \tilde{T}$ has its minimum possible value. For flow over an obstacle (Fig. 3), the sill states have relatively shallow lower layers and are therefore confined to the right branch of the curve. States corresponding to flow through a pure contraction (Fig. 2) exist on both branches. Maximal exchange for this case corresponds to the bottom of the curve.
In the Stommel and Farmer (1953) model, the estuary has a fresh and relatively thin surface layer, fed by river runoff. Mixing between this layer and the underlying salty water (which intrudes from the ocean) is imagined to be due to tides and winds and is therefore imposed independently of the state of flow. If there is no mixing, the fresh layer simply passes through the estuary and out through the strait, where a hydraulic control is supposed to exist. The corresponding state lies high up on the left-hand branch of the corresponding $U$ curve. If the mixing is increased, the salinity difference between layers (analogous to our $\Delta \tilde{T}$ ) decreases and the corresponding state lies lower and to the right on the $\mathrm{U}$ curve. If the mixing is sufficiently strong, the minimum in the curve will be reached and the exchange will therefore become maximal. An increase in mixing beyond this point can have no further effect on the exchange flow, provided that steady twolayer conditions are preserved. A further decrease in salinity would require a volume exchange past the limit imposed by the hydraulic constraint to conserve salt. The estuary in the case of mixing past the maximal exchange limit is said to be overmixed. The nature of the overmixed state depends on the estuary geometry and may not be obvious in advance. Whitehead et al. (2003) present a laboratory example that shows how forcing of the estuary past the threshold value results in an increase in mechanical mixing without a change in the layer buoyancy difference.

Our thermally driven model departs from this picture in several ways. First, the constriction may be due to a topographic sill rather than to a pure width contraction. In such cases, controlled solutions have a relatively thick upper layer over the sill $\left(h_{1} / H>0.5\right.$; Fig. 3) and solutions are restricted to the right branch of the $U$ curve. Moreover, the maximal exchange limit for such cases is reached not at the minimum of the curve but at a point slightly to the right. The threshold value of $h_{1} / H$ depends on the sill depth $H$ relative to the depth $H_{\mathrm{ms}}$ of the marginal sea and can be calculated as shown in appendix B. For a vanishingly small obstacle $\left(H / H_{\mathrm{ms}} \rightarrow\right.$ 1 ), maximal exchange occurs at $h_{1} / H=0.5$ (and $\Delta \tilde{T}=$ 2.52), whereas an obstacle occupying nearly the full water column $\left(H / H_{\mathrm{ms}} \rightarrow 0\right)$ exercises maximal exchange at $h_{1} / H=0.625$ (and $\Delta \tilde{T}=2.85$ ). The range of values spanning these limits is indicated by the short, thickest portion of the U curve in Fig. 4.

Another significant departure lies in the nature of the forcing. We impose a surface heat loss $Q$ over the marginal sea and, although a larger $Q$ presumably leads to a higher level of internal mixing, the mixing itself is not imposed. As demonstrated by Finnigan and Ivey (2000) 


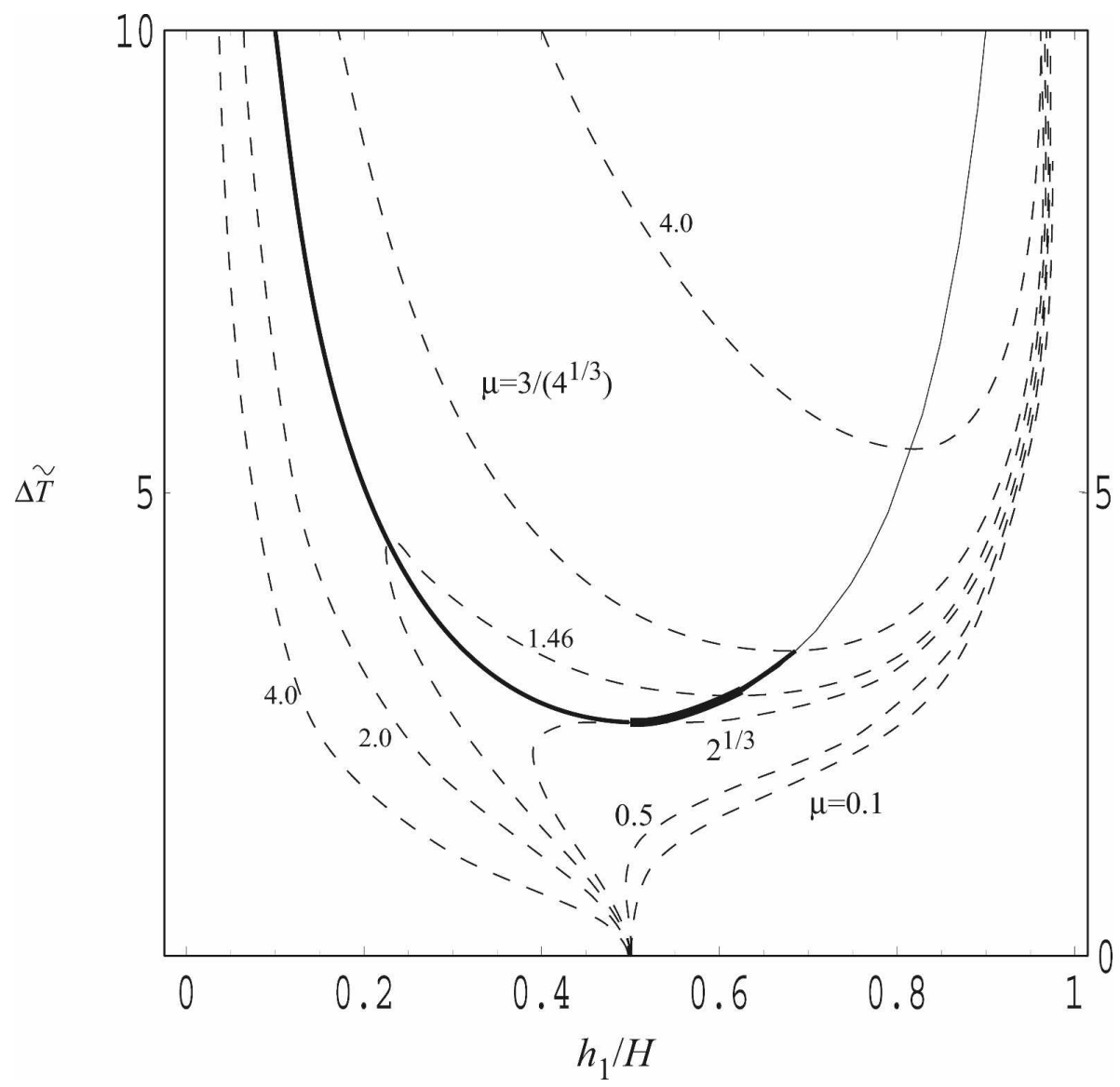

FIG. 4. The solid U curve, based on Eq. (10), gives the nondimensional temperature difference as a function of the upper-layer thickness at the most constricted section of the strait (either a narrows or a sill). The thickest portion of this curve gives the range $0.5 \leq h_{1} / H \leq$ 0.625 of possible maximal exchange states, ranging from an obstacle of zero height (a pure contraction) to an obstacle that occupies the full water depth. The dashed contours give values of the forcing parameter $\mu$. Some of these contours cross the $U$ curve twice, indicating possible multiple states for the same forcing. The thickened portions of the $\mathrm{U}$ curve indicate the range over which this is possible.

in a laboratory experiment in a semienclosed, thermally driven, nonrotating basin, an increase in $Q$ does not necessarily move the system toward a maximal exchange state. Moreover, the nature of maximal exchange state, if reached, is apt to be quite different. Although the layer depths at the constriction become locked in to the threshold values indicated above, the volume exchange and temperature difference need not be. An increase in $Q$ must always result in a larger heat flux through the strait, and this must be carried by a larger volume flux and/or a larger temperature difference, provided the two-layer character of the system is preserved. The notion of overmixing therefore seems less relevant.

What now remains to be determined is where on the $\mathrm{U}$ curve the solution for a particular $Q$ lies and whether, in fact, stronger $Q$ causes the flow state to tend toward maximal conditions. As it stands, the curve only gives a relationship between $h_{1} / H, T_{1}-T_{2}$, and $Q$ (which is hidden in the scaling for the temperature difference).

\section{d. Relating the forcing to the solution}

We now attempt to establish an independent relation between $h_{1} / H$ and $\Delta \tilde{T}$. One approach is based on the expectation that the surface inflow into the marginal sea forms a boundary current whose width scales with the Rossby radius of deformation (Fig. 1a, inset). If the thermal wind relation holds for this current, and if the motion of the underlying fluid can be neglected, then the characteristic boundary layer depth $H_{b}$, width $L$, and velocity $V_{b}$ are related by (4) and (5). All assumptions are supported by numerical simulations to follow. Conservation of the volume flux and energy between the most constricted section of the strait and the beginning of the boundary current (where $H_{b}, L$, and $V_{b}$ are 
measured) is then sufficient to provide the new relation between $h_{1} / H$ and $\Delta \tilde{T}$.

Conservation of energy is enforced along the wall streamline that runs from the most constricted section to the section marking the formation of the boundary current. This step assumes that no hydraulic jump is present between the two sections because such a feature would generally lead to dissipation of energy. Solutions that qualify include all of the submaximal cases for flow over an obstacle (Fig. 3) as well as those shown in Figs. $2 \mathrm{~b}-\mathrm{d}$ for flow through a contraction. All have relatively thick upper layers at the most constricted section and all block information generated in the open ocean from entering the marginal sea. Solutions that do not qualify include all maximal solutions with jumps at both ends of the strait as well as the submaximal solutions of Figs. 2f-h. However, it is possible to extend the theory to the latter submaximal solutions by enforcing conservation of energy between the most constricted section and the exterior ocean because no jump exists within this interval. If, in fact, the inflowing surface layer approaches the strait as a geostrophic boundary layer with the same properties as those assumed for the marginal sea, and along the same wall, then the calculation becomes identical and the theory can be applied to all submaximal solutions. In the numerical model simulations described later, the inflow does not, in fact, approach the strait as a boundary layer but rather as a broad, roughly uniform flow. The theory must then be modified as described in section 2e.

Because the Bernoulli Eq. (8) contains the quantities $V_{1}, H_{b}$, and $V_{b}$, the object is to get expressions for each in terms of the temperature difference and $h_{1} / H$. One can start with Eq. (2) and use the upper-layer continuity relation (7) and the thermal wind relation (4) to obtain

$$
\frac{A Q}{\rho_{o} c_{p}}=V_{b} H_{b} L\left(T_{1}-T_{2}\right)=\frac{\alpha g H_{b}^{2}}{2 \rho_{o} f}\left(T_{1}-T_{2}\right)^{2}
$$

or

$$
H_{b}^{2}=\frac{2 A Q f \alpha}{g \rho_{0}^{2} c_{p}}\left(\frac{\rho_{o}}{\alpha\left(T_{1}-T_{2}\right)}\right)^{2} .
$$

Next, use the thermal wind relation (4) along with the approximation (5) for $L$ and (12) to obtain

$$
V_{b}^{2}=\left(\frac{2 g Q A f \alpha}{\rho_{o}^{2} c_{p}}\right)^{1 / 2} \kappa^{-2} \text {. }
$$

Finally, rearrange (2) as

$$
V_{1}=\frac{A Q}{\rho_{o} W c_{p} h_{1}\left(T_{1}-T_{2}\right)}
$$

or

$$
V_{1}^{2}=\left(\frac{A Q \alpha}{W \rho_{o}^{2} c_{p} H}\right)^{2}\left(\frac{h_{1}}{H}\right)^{-2}\left[\frac{\alpha}{\rho_{o}}\left(T_{1}-T_{2}\right)\right]^{-2} .
$$

Now consider the Bernoulli Eq. (8), which can be rewritten as

$$
\frac{V_{1}^{2}}{2}\left[1-\frac{h_{1}^{2}}{\left(H-h_{1}\right)^{2}}\right]=g^{\prime}\left(H_{b}-h_{1}\right)+\frac{V_{b}^{2}}{2},
$$

if the condition of zero net flux $V_{1} h_{1}=V_{2}\left(H-h_{2}\right)$ is used. Substitution of the above expressions for $H_{b}$, $V H_{b}^{2}$, and $V_{1}^{2}$ then lead to

$$
\frac{h_{1}}{H} \Delta \tilde{T}^{3}-\mu \Delta \tilde{T}^{2}+\frac{1}{2}\left(\frac{h_{1}}{H}\right)^{-2}\left\{1-\frac{\left(h_{1} / H\right)^{2}}{\left[1-\left(h_{1} / H\right)\right]^{2}}\right\}=0 \text {, }
$$

where

$$
\mu=\frac{2^{1 / 2} W^{2 / 3} f^{1 / 2} \rho_{o}^{1 / 3} c_{p}^{1 / 6}}{(g A Q \alpha)^{1 / 6}}\left(1+\frac{1}{2} \kappa^{-2}\right) .
$$

Equation (16) gives an independent relation between $\Delta \tilde{T}$ and $h_{1} / H$ that follows from the requirement that the marginal sea inflow forms a geostrophic boundary current while conserving volume flux and wall energy. It forms a kind of matching condition between two regions with different dynamics: the strait and the marginal sea. Together with (10), which is based on the critical condition and the heat budget for the marginal sea and which results in the U curve, (16) determines the values of $\Delta \tilde{T}$ and $h_{1} / H$ for a given forcing. Valid solutions are formally restricted to the right branch of the $U$ curve but can be extended to the left branch if the ocean flow entering the strait is contained in a geostrophic boundary layer with the same properties (i.e., the same $\kappa)$ as for the marginal sea boundary layer. The forcing is specified by the parameter $\mu$, which decreases as the heat loss $Q$ increases or the strait width $W$ decreases. Thus, small values of $\mu$ are associated with more highly constricted outlets and stronger thermal forcing.

A selection of dashed contours of constant $\mu$ appears in Fig. 4 along with the U curve. Intersections between the two give the values of $\Delta \tilde{T}$ and $h_{1} / H$ corresponding to a given forcing $\mu$. Dashed contours with lower values of $\mu$ intersect the curve at lower values of $\Delta \tilde{T}$, suggesting that stronger forcing or stronger choking pushes the state toward the maximal exchange limit, as expected. The threshold value $\mu_{m}$ for maximal exchange depends on the relative sill depth $H / H_{\mathrm{ms}}$. In the case of a pure contraction $\left(H / H_{\mathrm{ms}}=1\right)$, where maximal conditions lie at the minimum of the U curve, Fig. 4 suggest $\mu_{m}=2^{1 / 3}$. For cases of obstacles with finite height $0 \leq H / H_{\mathrm{ms}}<1$, 
the value of $\mu$ is slightly greater. If $\mu$ falls below $\mu_{m}$ as a result of an increase in the heat flux $Q$, say, then Eq. (16) presumably breaks down due to the presence of a hydraulic jump on either side of the most constricted section. Because (10) remains valid, $h_{1} / H$ and $\Delta \tilde{T}$ must remain at their threshold values for maximal flow. Let $\Delta \tilde{T}_{m}$ denote the threshold value of $\Delta \tilde{T}$, so that

$$
T_{1}-T_{2}=\left(\frac{A^{2} Q^{2}}{\alpha g W^{2} \rho_{o} c_{p}^{2}}\right)^{1 / 3} \frac{\Delta \tilde{T}_{m}}{H}
$$

in view of (11). It follows from (9) that

$$
Q_{v}=\left(\frac{A Q \alpha g W^{2}}{\rho_{o}^{2} c_{p}}\right)^{1 / 3} \frac{H}{\Delta \tilde{T}_{m}} .
$$

Thus, the temperature difference and volume flux increase in proportion to $Q^{2 / 3}$ and $Q^{1 / 3}$ once the exchange becomes maximal.

As suggested in Fig. 4, and by the above comments, the solutions can be divided into several categories depending on the value of $\mu$.

1) $\mu>3 /\left(4^{1 / 3}\right)$ : WEAK FORCING

The corresponding curves in Fig. 4 are separated into two distinct branches, as shown, for example, by $\mu=$ 4.0. The lower branch, which lies to the left of the figure, never intersects the $U$ curve. The upper branch intersects the $U$ curve once and only along its righthand branch. The corresponding states are submaximal and uniquely determined, and they have a relatively deep upper layer in the strait $\left(h_{1} / H>0.5\right)$.

2) $2^{1 / 3}<\mu<3 /\left(4^{1 / 3}\right)($ OR $1.26<\mu<1.89)$ : MODERATE FORCING

There is now a single dashed curve for each $\mu$, each beginning at $h_{1} / H=0.5$ along the lower axis. The curves initially climb to the left before they swing back to the right. At its zenith, each curve crosses the left branch of the U curve; it then descends and swings back to the right, intersecting the right branch. Two possible basin states now formally exist for the same forcing. The left branch root has relatively large $\Delta \tilde{T}$, weak volume exchange, and a relatively small upper-layer depth (and large velocity) in the strait. The multiple states live on the portion of the $U$ curve that has been moderately thickened in Fig. 4. Note also that the possible maximal states, indicated by a heavily thickened interval of the same curve (and corresponding to $2^{1 / 3} \leq \mu \leq 1.67$ ), also have a multiple state partner on the left branch.

3) $\mu=\mu_{m} 2^{1 / 3} \leq \mu_{m} \leq 1.46$ : LIMITS OF MAXIMAL EXCHANGE

For a pure contraction, the relevant contour is $\mu_{m}=$ $2^{1 / 3}$, which begins at the middle point of the lower axis and makes grazing contact with the minimum of the solid curve. The layer thicknesses in the contraction are equal. If an obstacle is present in the strait, the limit of maximal exchange lies along the U curve slightly to the right of the minimum value and within the thick dashed portion.

\section{4) $\mu<\mu_{m}$ : FORCING PAST THE MAXIMAL THRESHOLD}

The curves, which again originate at $h_{1} / H=0.5$ on the lower axis, either do not intersect the $U$ curve or do so at a point to the left of the maximal exchange solution. Equation (16) is no longer valid. The layer thicknesses at the most constricted section remain at their values for maximal exchange, and the dimensional temperature difference and volume flux obey (18) and (19).

For the assumed strait geometry (consisting of either a straight channel with a sill or a smoothly contracting channel with a flat bottom), solutions along the left branch of the $U$ curve are possible only for the flatbottom case. Thus, it would appear that multiple states are possible only in this case.

\section{$e$. The case of a relatively small upper-layer depth in the strait}

In the numerical results discussed below, the temperature of the inflowing layer is established by restoring it to a certain value over a vertical thickness $H_{o}$ and over a broad horizontal area of the ocean outside the mouth of the marginal sea. The associated upper-layer inflow tends to be uniformly distributed in the horizontal rather than concentrated in a boundary layer. A theory for flow states lying on the left branch of the $U$ curve can then be developed by approximating these conditions. In particular, one can require that the ocean be quiescent away from the mouth of the strait and that the upper layer has a uniform thickness $H_{o}$. (This is consistent with the picture normally assumed in classical hydraulics without rotation.) Then the Bernoulli Eq. (15) reads

$$
\frac{V_{1}^{2}}{2}\left[1-\frac{h_{1}^{2}}{\left(H-h_{1}\right)^{2}}\right]=g^{\prime}\left(H_{o}-h_{1}\right)
$$

Use of (6) and (14) then lead to

$$
\Delta \tilde{T}^{3}=\frac{\left\{1-\frac{\left(h_{1} / H\right)^{2}}{\left[1-\left(h_{1} / H\right)\right]^{2}}\right\}}{2\left(\frac{h_{1}}{H}\right)^{2}\left(\frac{H_{o}}{H}-\frac{h_{1}}{H}\right)} .
$$


For solutions on the left-hand branch of the U curve $\left(h_{1} / H<0.5\right)$ the left-hand side of $(20)$ is positive, and thus $h_{1}<H_{o c}$. Equation (21) then guarantees that $\Delta \tilde{T}$ is positive.

If $\Delta \tilde{T}^{3}$ is eliminated between (21) and (10), the following expression for the ocean layer thickness is obtained:

$$
\frac{H_{o}}{H}=\frac{h_{1}}{H}+\frac{\frac{1}{\left(h_{1} / H\right)^{2}}-\frac{1}{\left(1-h_{1} / H\right)^{2}}}{2\left[\frac{1}{\left(h_{1} / H\right)^{3}}+\frac{1}{\left(1-h_{1} / H\right)^{3}}\right]} .
$$

We have assumed conservation of energy, and therefore an absence of hydraulic jumps, between the open ocean and the most constricted section. As suggested in Fig. 2, the solutions are then valid for $H_{o} / H<0.5$. In the strait itself, the upper-layer thickness will be $<H_{o}$ and less than the lower-layer thickness. It is important to note that the parameter $\mu$ no longer appears and that $H_{o} / H$ is now regarded as an observable, a result consistent with the passage of information into the marginal sea from the open ocean, not the reverse. The heat loss $Q$ over the marginal sea only appears as a scale factor (11) for the temperature difference.

\section{Numerical simulations}

The theory outlined in the preceding section provides estimates of the exchange rate, layer thickness, and temperature anomaly for hydraulically controlled exchange flows between two basins. There are several key assumptions required for these derivations, many being common for hydraulic control theories. The novel aspect of the present approach, which allows for the determination of unique hydraulically controlled states given the forcing and geometry, is the connection between the flow in the strait and the flow in the interior of the two basins. It is asserted that the dynamics that govern the balances within the marginal sea are essentially independent of the hydraulic control within the strait. In this section, a high-resolution general circulation model, which resolves the dynamics both within the strait and within the two semienclosed basins, is used to test the basic predictions of the theory.

\section{a. Model configuration}

The numerical model used is the Massachusetts Institute of Technology (MIT) general circulation model (Marshall et al. 1997). This model solves the hydrostatic, primitive equations on a uniform, staggered Cartesian C-grid with level vertical coordinates. A partial cell treatment of the bottom topography is accurate for steep topography in the presence of stratification, expected to be important for the present problem.

The model domain consists of two semienclosed basins connected by a narrow strait. In one set of calculations, the basins are of uniform depth of $100 \mathrm{~m}$ and the strait is of uniform width, with a 50-m-deep sill at the midpoint (Figs. 5a, 7). In another set of calculations, the two semienclosed basins and the strait are of uniform depth of $50 \mathrm{~m}$ and the strait narrows gradually to the midpoint (Fig. 5b). The width of the strait for the cases with a sill is nominally $2 \mathrm{~km}$, although this is varied. The strait is $1.2 \mathrm{~km}$ at its narrowest point for most of the calculations with the lateral constriction. A summary of the model configurations and forcing is given in Table 1.

The model is forced by cooling one basin, called the marginal sea, with a uniform heat loss. The temperature within the other basin, called the open ocean, is restored toward $10^{\circ} \mathrm{C}$ from the surface to a depth of $H_{o}$ with a time scale of 2 days. There is no forcing within the strait. This system produces an exchange between the two basins through which, in equilibrium, the heat flux into the marginal sea balances the surface cooling (which is specified). The restoring of temperature in the open ocean provides the required heat. Note that neither the exchange rate nor the temperature of the water flowing out of the marginal sea is specified, but each is instead determined by the strait and marginal sea dynamics.

The domain is $40 \mathrm{~km}$ wide ( $x$ direction) and $200 \mathrm{~km}$ long ( $y$ direction). The horizontal resolution is variable, ranging in $x$ from $2 \mathrm{~km}(0-30 \mathrm{~km})$ to $1 \mathrm{~km}(30-36 \mathrm{~km})$ to $400 \mathrm{~m}(36-40 \mathrm{~km})$. The horizontal resolution is also variable in $y$, ranging from $1 \mathrm{~km}(0-30 \mathrm{~km})$ to $400 \mathrm{~m}$ $(30-50 \mathrm{~km})$ to $1 \mathrm{~km}(50-60 \mathrm{~km})$ to $2 \mathrm{~km}(60-200 \mathrm{~km})$. The spatial resolution within the strait is uniform in both the $x$ and $y$ directions at $400 \mathrm{~m}$. There are 25 uniformly spaced levels in the vertical. For the calculations in the sill domain, the vertical resolution is $4 \mathrm{~m}$, while for the lateral constriction cases the vertical resolution is $2 \mathrm{~m}$.

The model incorporates second-order vertical viscosity and diffusivity with coefficients $10^{-5} \mathrm{~m}^{2} \mathrm{~s}^{-1}$. The vertical diffusion is increased to $1000 \mathrm{~m}^{2} \mathrm{~s}^{-1}$ for statically unstable conditions to represent vertical convection. Horizontal viscosity is parameterized as a secondorder operator with the coefficient $A_{h}$ determined by a Smagorinsky (1963) closure as

$$
A_{h}=\left(\nu_{s} / \pi\right)^{2} L^{2} D
$$

where $v_{s}$ is a nondimensional coefficient taken to be 2 here, $L$ is the grid spacing, and $D$ is the deformation rate, defined as 


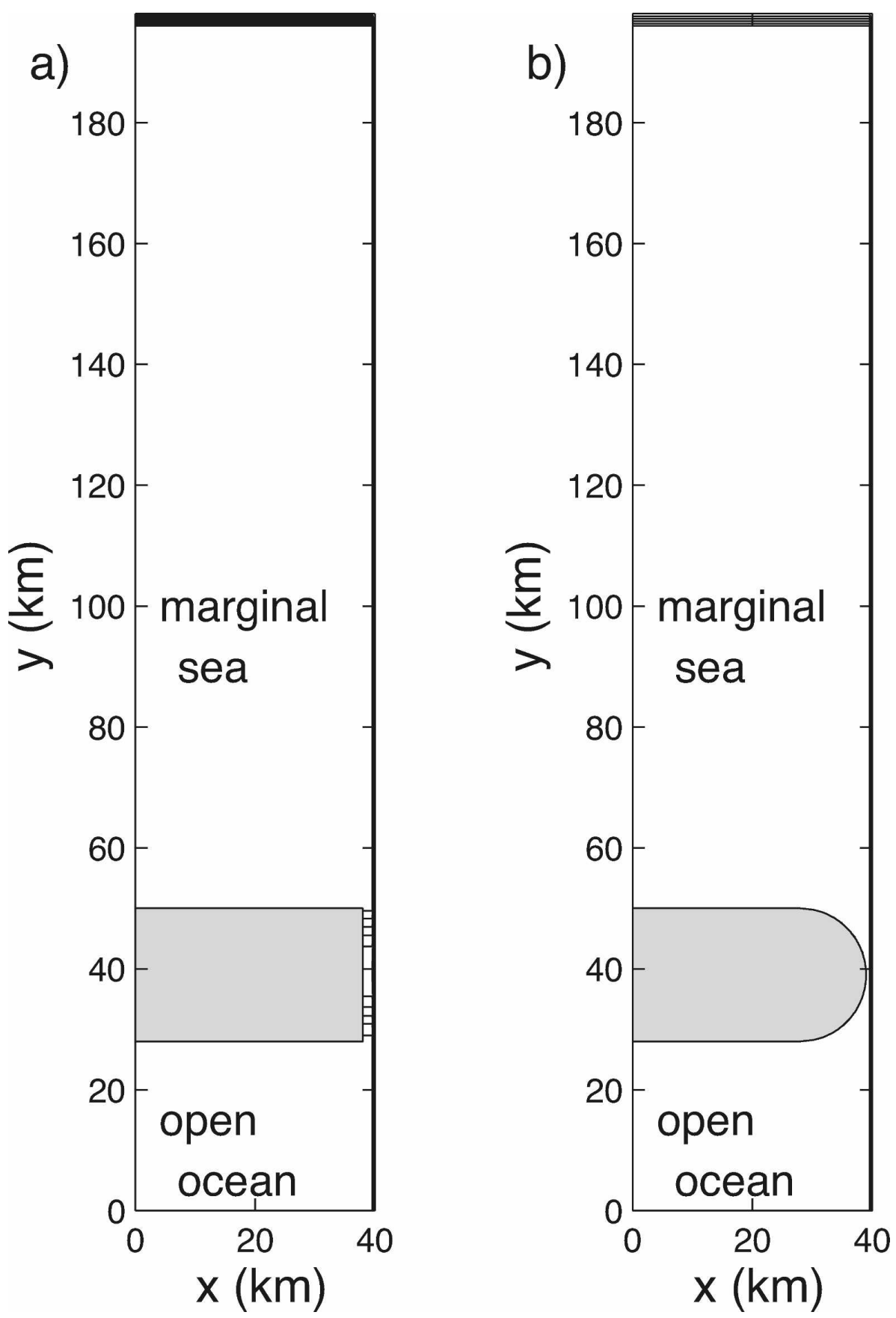

FIG. 5. Model domain configuration for two cases: (a) topographic sill and (b) lateral constriction.

$$
D=\left[\left(u_{x}-v_{y}\right)^{2}+\left(u_{y}+v_{x}\right)^{2}\right]^{1 / 2}
$$

where $u$ and $v$ are the resolved horizontal velocities and subscripts indicate partial differentiation. The lateral boundary conditions are no normal flow and no stress for momentum (free slip) and no normal heat flux for temperature.

The model calculates density from a linear equation of state as $\rho=\rho_{\mathrm{o}}-\alpha T$, where $\rho_{\mathrm{o}}=0.2 \mathrm{~kg} \mathrm{~m}^{-3}{ }^{\circ} \mathrm{C}^{-1}$ is the thermal expansion coefficient and $\rho_{\mathrm{o}}=1026.5 \mathrm{~kg}$ $\mathrm{m}^{-3}$ is a reference density.

The model is run for a period of 720 days. It takes about 400 days for the properties of the exchange flow to equilibrate, although there can be considerable highfrequency variability present within the strait. The analysis in this section is taken from temporal averages over the final 180 days of integration. The standard calculations apply a uniform cooling over the marginal sea. 
TABLE 1. Summary of model runs with key parameters. Runs S1-S10 are configured with a sill and a uniform width strait, while runs $\mathrm{C} 1-\mathrm{C} 9$ have a flat bottom and a variable width strait.

\begin{tabular}{lcccccc}
\hline \hline Run & $Q\left(\mathrm{~W} \mathrm{~m}^{-2}\right)$ & $f_{0}\left(10^{-4} \mathrm{~s}^{-1}\right)$ & $W(\mathrm{~m})$ & $\mu$ & $H_{o}(\mathrm{~m})$ & $h_{1} / H$ \\
\hline S1 & 360 & 1 & 3600 & 3.19 & & 0.77 \\
S2 & 720 & 1 & 2000 & 1.92 & & 0.69 \\
S3 & 720 & 1 & 3600 & 2.84 & & 0.78 \\
S4 & 1080 & 1 & 2000 & 1.80 & & 0.71 \\
S5 & 540 & 1 & 2000 & 2.02 & & 0.72 \\
S6 & 120 & 1 & 3600 & 3.83 & & 0.80 \\
S7 & 720 & 1.5 & 2000 & 2.35 & & 0.72 \\
S8 & 720 & 1 & 1200 & 1.37 & & 0.64 \\
S9 & 720 & 0.5 & 2000 & 1.36 & & 0.65 \\
S10 & 720 & 0.5 & 1200 & 0.97 & & 0.64 \\
C1 & 720 & 1 & 1200 & & 19 & 0.23 \\
C2 & 720 & 2 & 1200 & & 10 & 0.11 \\
C3 & 720 & 1 & 1200 & & 5 & 0.08 \\
C4 & 720 & 1 & 1200 & & 16 & 0.19 \\
C5 & 720 & 1 & 1200 & & 10 & 0.12 \\
C6 & 720 & 1 & 1200 & & 22 & 0.24 \\
C7 & 720 & 1 & 1200 & & 24 & 0.28 \\
C8 & 720 & 1 & 1200 & & 28 & 0.30 \\
C9 & 720 & 1 & 2000 & & 10 & 0.12 \\
\hline
\end{tabular}

\section{b. Circulation with sill-induced hydraulic control}

The horizontal velocity and temperature at $2-\mathrm{m}$ depth, averaged over the final 180 days of integration, for a case with a sill and a cooling of $360 \mathrm{~W} \mathrm{~m}^{-2}$ (run S1) are shown in Fig. 6. The warm water of the open ocean basin flows into the marginal sea and forms a cyclonic boundary current that encircles the basin. The temperature within the boundary current decreases as it flows into the marginal sea and travels around the basin. The coldest waters, found in the middle of the marginal sea at the surface, spill over the sill and back into the open ocean. The width of the cyclonic current in the marginal sea is on the order of the internal deformation radius and wider than the width of the strait. The flow from the open ocean into the strait is not carried in a boundary current but instead is fed by a weak basin-scale recirculation.

The mean along-strait temperature and velocity taken along a section through the strait in the vicinity of the sill is shown in Fig. 7. The exchange flow is well characterized by a two-layer system, and the strait is sufficiently narrow such that the flow is independent of the cross-strait position. The source waters in the open ocean are of nearly uniform temperature and occupy most of the water column. In the vicinity of the sill, the inflowing water is thicker than the outflowing water and the interface marking the transition between the inflowing and outflowing waters is distinct. Quantities such as the composite Froude number $G^{2}$ [see Eqs. (1) and (6)] can be calculated based on time-average and

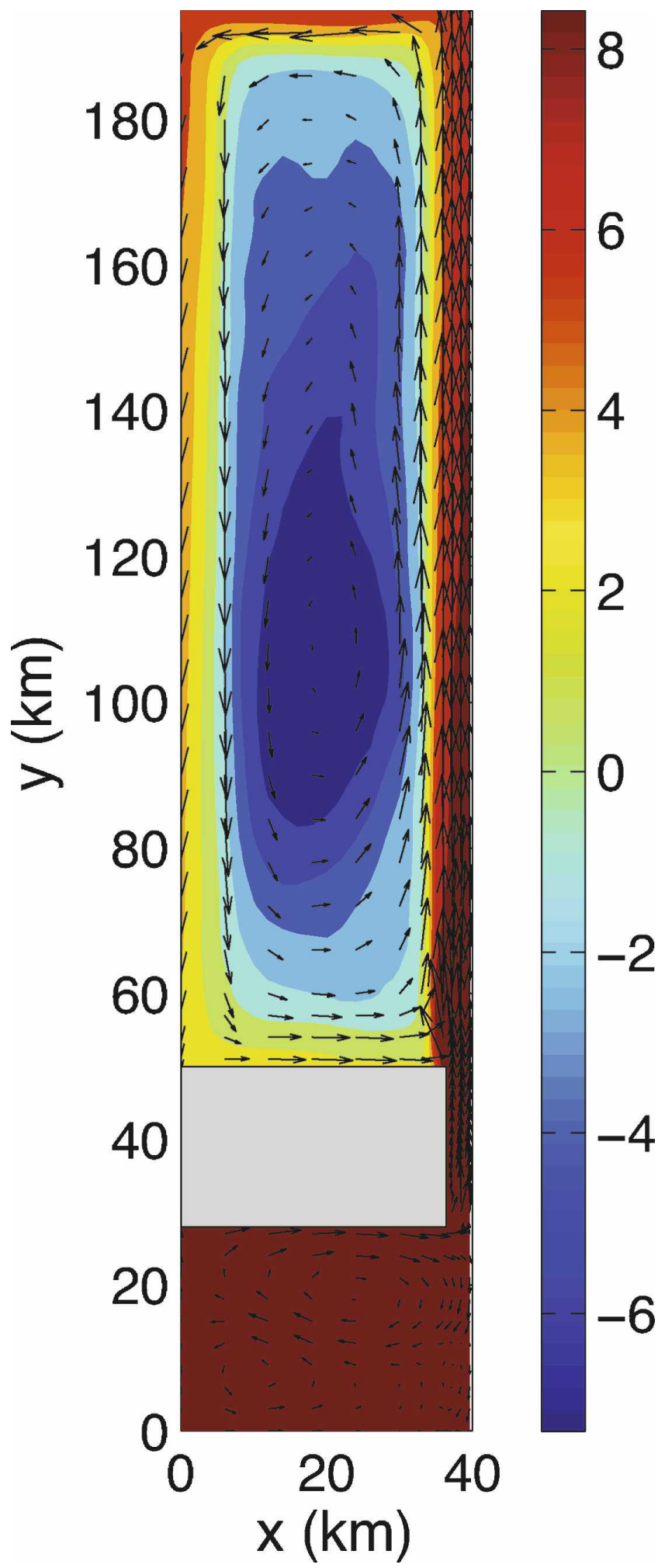

FIG. 6. Mean horizontal velocity (every third grid point) and temperature at 2-m depth for the std case with a sill (run S1).

layer-average velocities and temperatures, the layer interface being defined by the depth of maximum stratification. As shown in Fig. 8, the so-determined $G^{2}$ becomes unity, indicating critical flow, slightly to the 
a)
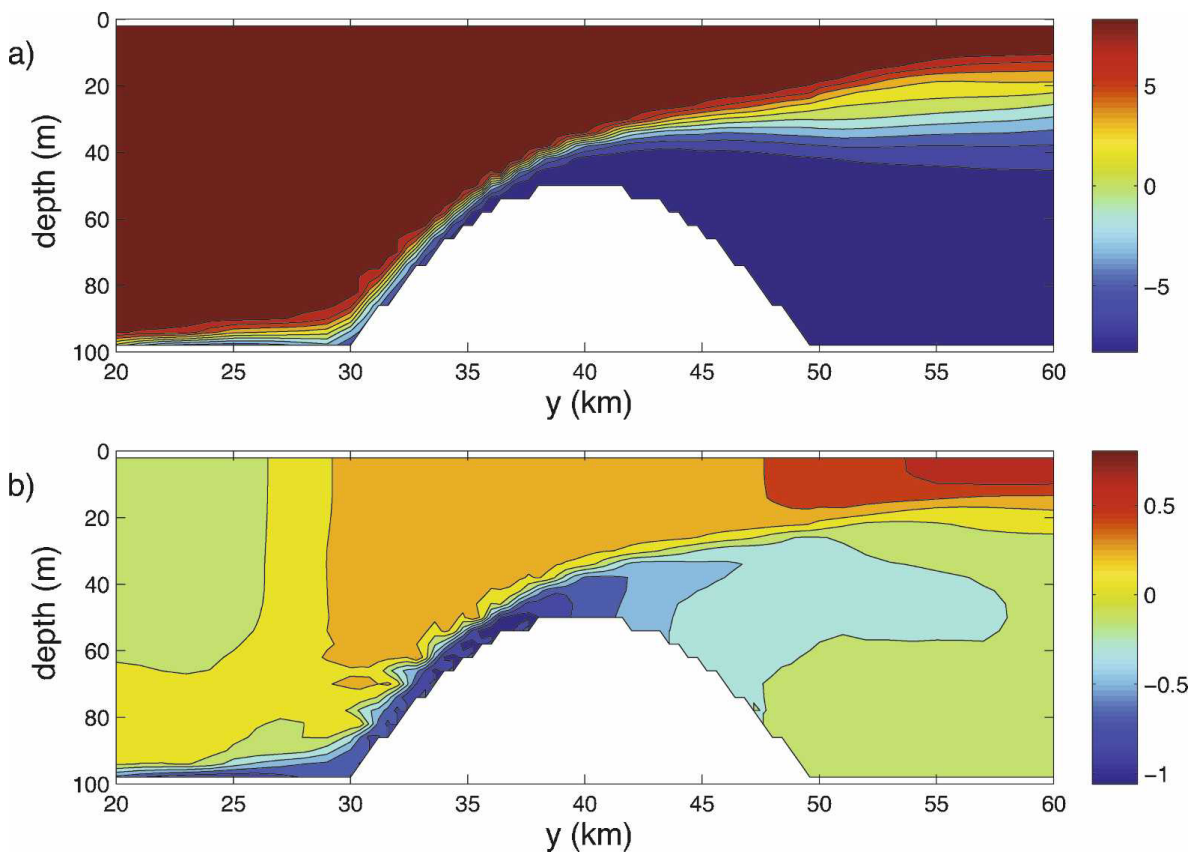

FIG. 7. Section of mean properties taken through the strait for (a) $T\left({ }^{\circ} \mathrm{C}\right)$ and (b) $v\left(\mathrm{~m} \mathrm{~s}^{-1}\right)$ for run $\mathrm{S} 1$.

right of the sill and is $>1$ to the left, as expected. As the inflowing waters enter the marginal sea, the upper layer continues to thin and the upper layer velocity increases, approaching $0.5 \mathrm{~m} \mathrm{~s}^{-1}$. The outflowing dense water layer becomes very thin just downstream of the sill and its velocity exceeds $1 \mathrm{~m} \mathrm{~s}^{-1}$. This density and velocity distribution is typical of hydraulically controlled exchange with a sill.

Our theory is based on the assumption that the internal Bernouli function $\Delta B$ [Eq. (8)] is conserved along the right-hand wall as the flow enters the marginal sea. As a test, the mean-based $\Delta B$ is evaluated from the model as a function of latitude through the strait and extending into the marginal sea, as shown in Fig. 8 . The value varies only weakly through the strait and between the strait and marginal sea (which begins at $50-\mathrm{km}$ latitude), generally supporting the theoretical premise.

The properties of the exchange at the sill location are shown in Fig. 9 as a function of time. These quantities are calculated at the model grid point closest to the right-hand boundary of the strait (facing the marginal sea) at $y=40 \mathrm{~km}$, the shallowest point of the sill. The right-hand side of the strait is chosen because it corresponds to the streamline along which energy is conserved following the theory. The velocities increase over the first several hundred days, after which they fluctuate weakly around $0.3 \mathrm{~m} \mathrm{~s}^{-1}$ inflow and $0.6 \mathrm{~m} \mathrm{~s}^{-1}$ outflow. The temperature of the inflowing waters is close to $10^{\circ} \mathrm{C}$, the value to which it is restored in the open ocean. The outflowing water temperature rapidly decreases over the first several hundred days and approaches an equilibrium value of around $-8^{\circ} \mathrm{C}$ (of course, it is only the temperature difference that is dynamically and thermodynamically significant). The value of $G^{2}$ shows variations between 0.9 and 1.3 , on time scales of 10-100 days. Values of $G^{2}$ that are closer toward the open ocean increase well above 1 , while values toward the marginal sea are always less than 1 ,

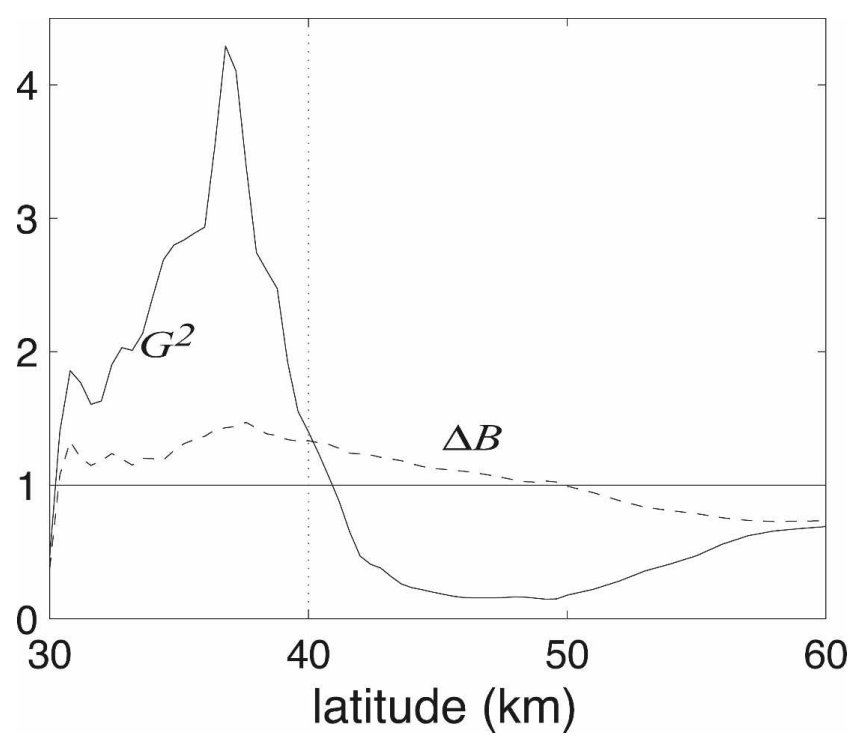

Fig. 8. Bernoulli function $\Delta B$ and the composite Froude number $G^{2}$ diagnosed from the model as a function of latitude through the strait $(30-50 \mathrm{~km})$ and into the marginal sea. 

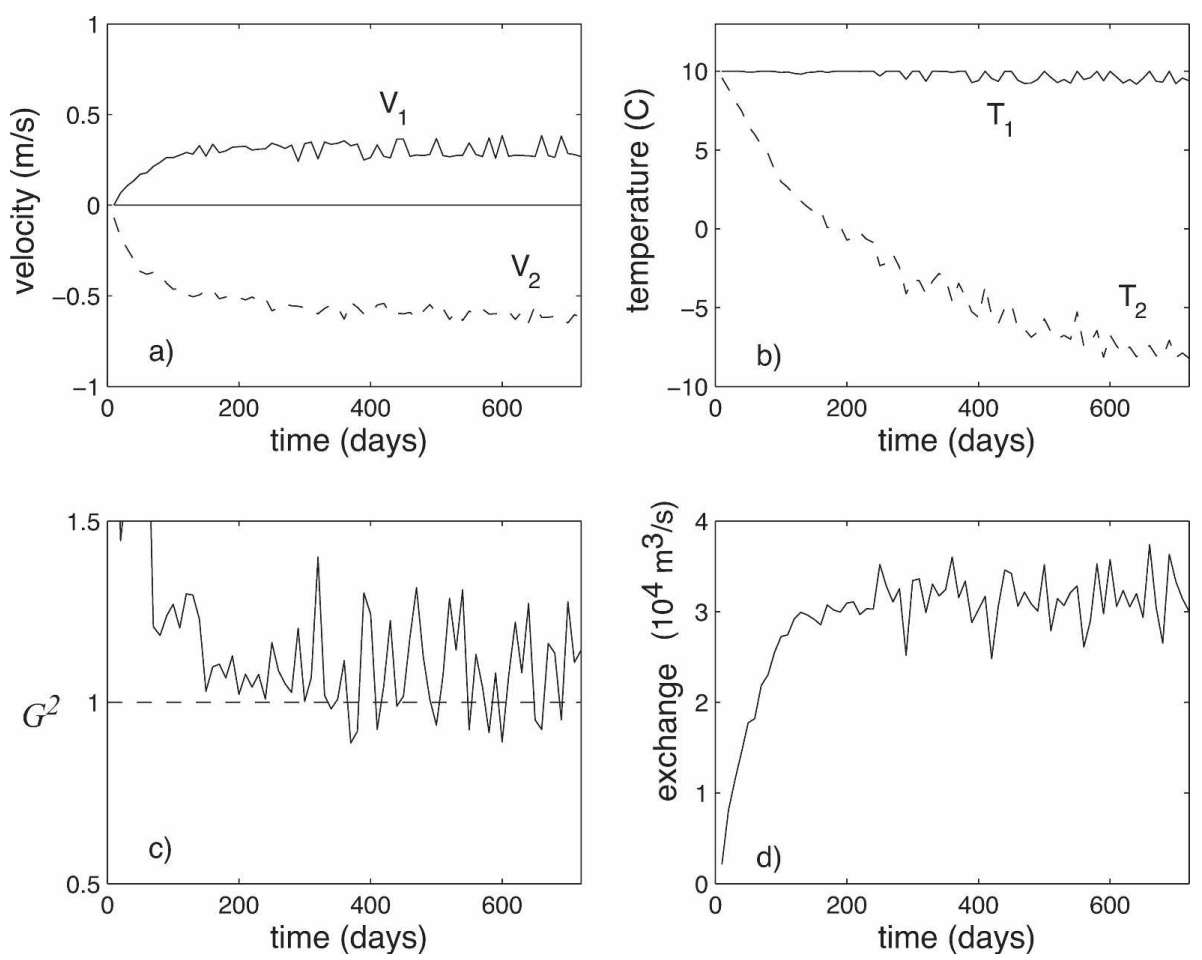

FIG. 9. Time series of (a) upper-layer (solid) and lower-layer (dashed) velocity, (b) upper-layer (solid) and lower-layer (dashed) $T$, (c) $G^{2}$, and (d) exchange rate for run S1.

consistent with the presence of a hydraulic control. The volume exchange settles into a pattern of $\pm 0.5 \mathrm{~Sv}$ $\left(1 \mathrm{~Sv} \equiv 10^{6} \mathrm{~m}^{3} \mathrm{~s}^{-1}\right)$ variability about a mean close to 3 $\mathrm{Sv}$, and on similar time scales.

\section{c. Constriction-induced hydraulic control}

The previous calculation with a topographic sill provides a configuration for which we expect the upperlayer thickness at the sill to be larger than the lowerlayer thickness, corresponding to the right-hand side of the theoretical $U$ curve. To access the left-hand side of the $U$ curve, we carry out calculations in which the hydraulic control is induced entirely by a lateral contraction of the strait width.

The horizontal velocity and temperature at 1-m depth, averaged over the final 180 days of integration, for a cooling of $720 \mathrm{~W} \mathrm{~m}^{-2}$ are shown in Fig. 10. The restoring in the open ocean is applied only to the upper $20 \mathrm{~m}$ of the water column. This provides an inflow thickness that is less than half the basin depth, as required to access the left-hand side of the $U$ curve in Fig. 4. Similar to the case with a sill, the warm water of the open ocean basin flows into the marginal sea and forms a cyclonic boundary current that encircles the basin. The temperature within the boundary current decreases as it flows into the marginal sea and travels around the basin. The coldest waters, found in the middle of the marginal sea, flow through the strait and back into the open ocean. The width of the cyclonic current in the marginal sea is on the order of the internal deformation radius, and it is much wider than the narrowest portion of the strait. Also, as found for the case with a sill, the flow from the open ocean into the strait is not carried in a boundary current but instead is fed by a weak basin-scale recirculation.

A section of temperature and velocity along the strait is shown in Fig. 11. The strait is sufficiently narrow that the properties are nearly uniform in the cross-strait direction. The average depth of maximum stratification for the inflowing waters is $19 \mathrm{~m}$, close to the restoring thickness in the open ocean. As the surface water enters the strait, the thickness decreases and the horizontal velocity increases. Just upstream of the narrowest portion of the strait, the thickness abruptly decreases and the velocity increases. The composite Froude number at this location is well below unity (Fig. 12), but it increases rapidly, crossing through the critical value near the narrowest section $(40 \mathrm{~km})$ and exceeding the critical value as the marginal sea is approached. The value of $\Delta B$ decreases only slightly through the strait, once again supporting the energy conservation constraint.

The properties of the exchange at the controlled location are shown in Fig. 13 as a function of time. The 


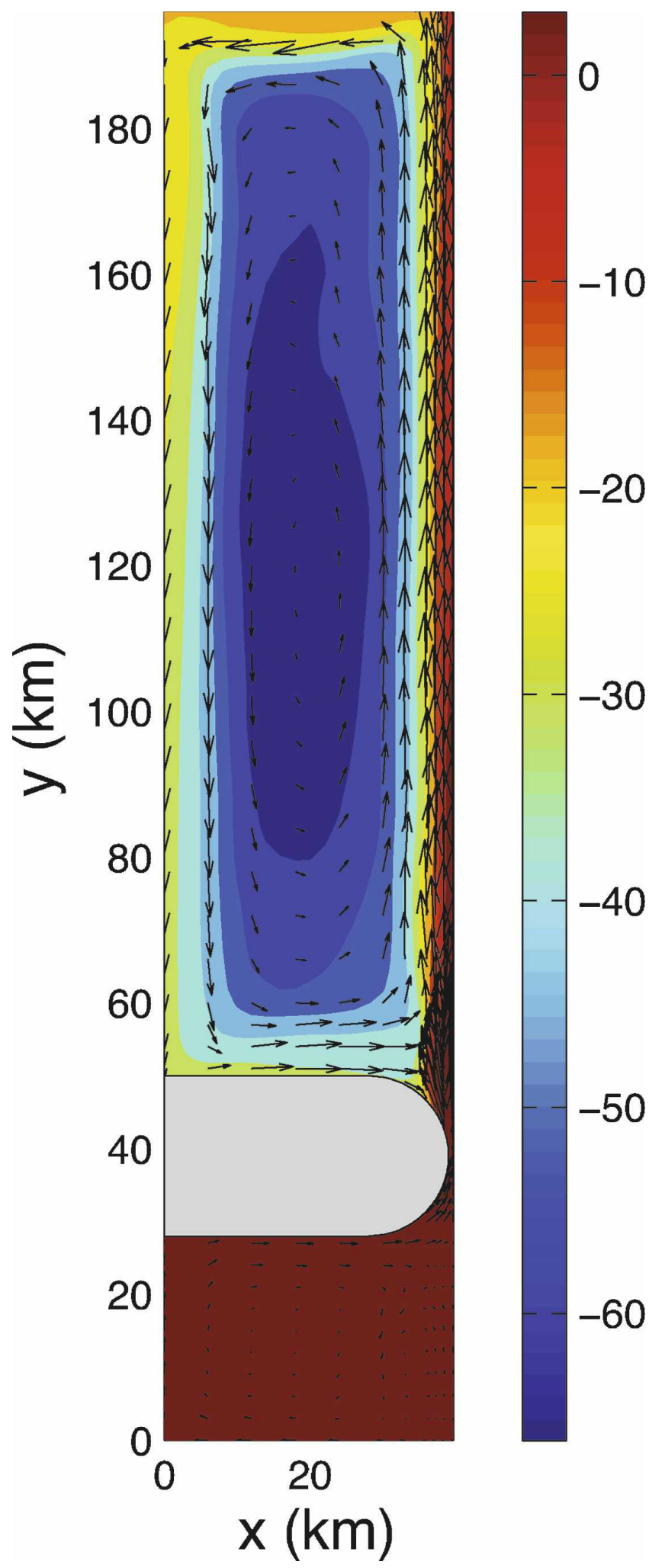

FIG. 10. Mean horizontal $v$ (every third grid point) and $T$ at 1-m depth for the std case with a lateral constriction (run C1). spinup time is again several hundred days, with the upper-layer velocity exceeding $1 \mathrm{~m} \mathrm{~s}^{-1}$ and the lowerlayer velocity approximately $0.3 \mathrm{~m} \mathrm{~s}^{-1}$. The temperature difference between the upper and lower layers is now larger, approximately $65^{\circ} \mathrm{C}$. The composite Froude number again fluctuates around the critical value of 1 .

\section{d. Comparison with theory}

The general circulation and exchange in each of the above model results are qualitatively consistent with what is expected based on the theory outlined in section 2 . The exchanges appear to be hydraulically controlled near the shallowest or narrowest part of the strait. The exchanges are well described by two layer systems with nearly homogeneous water masses entering and exiting the marginal sea. The circulation within the marginal sea is dominated by a cyclonic boundary current of width on the order of the internal deformation radius, and much wider than the strait. Comparisons are now made with the theory to both seek quantitative agreement and to determine if the more fundamental transitions in the flow structure expected from the theory are found in the model.

One of the most basic quantities describing the exchange between a marginal sea subject to buoyancy forcing and the open ocean is the temperature (or more generally density) anomaly generated within the marginal sea, as well as the volume outflow. Knowing the strength of the buoyancy forcing is not sufficient to provide a unique solution. For example, one could satisfy a given heat loss within the marginal sea by exchanging either a large amount of water with a relatively small temperature difference or a small amount of water with a large temperature difference. Some additional dynamical constraints must be taken into consideration. For the case of a wide and deep strait, such that a hydraulic control is not supported, Spall (2004) was able to relate the temperature difference between the inflowing and outflowing water masses to the basin geometry and surface forcing as

$$
\Delta T_{\mathrm{nh}}=\left(\frac{A Q f_{0}}{H_{\mathrm{o}}^{2} \alpha g C_{p}}\right)^{1 / 2} .
$$

This relation is based on some of the same constraints listed in section 2 and on the assumption that the upperlayer thickness imposed by temperature restoration in the open ocean $\left(H_{o}\right)$ is also the upper-layer thickness in the connecting strait. A similar relationship can be derived for marginal seas with a sill in the absence of hydraulic control (Iovino et al. 2008).

A series of calculations has been carried out in which the model parameters are varied, as summarized in Table 1. For the cases with a topographic sill, the 
a)
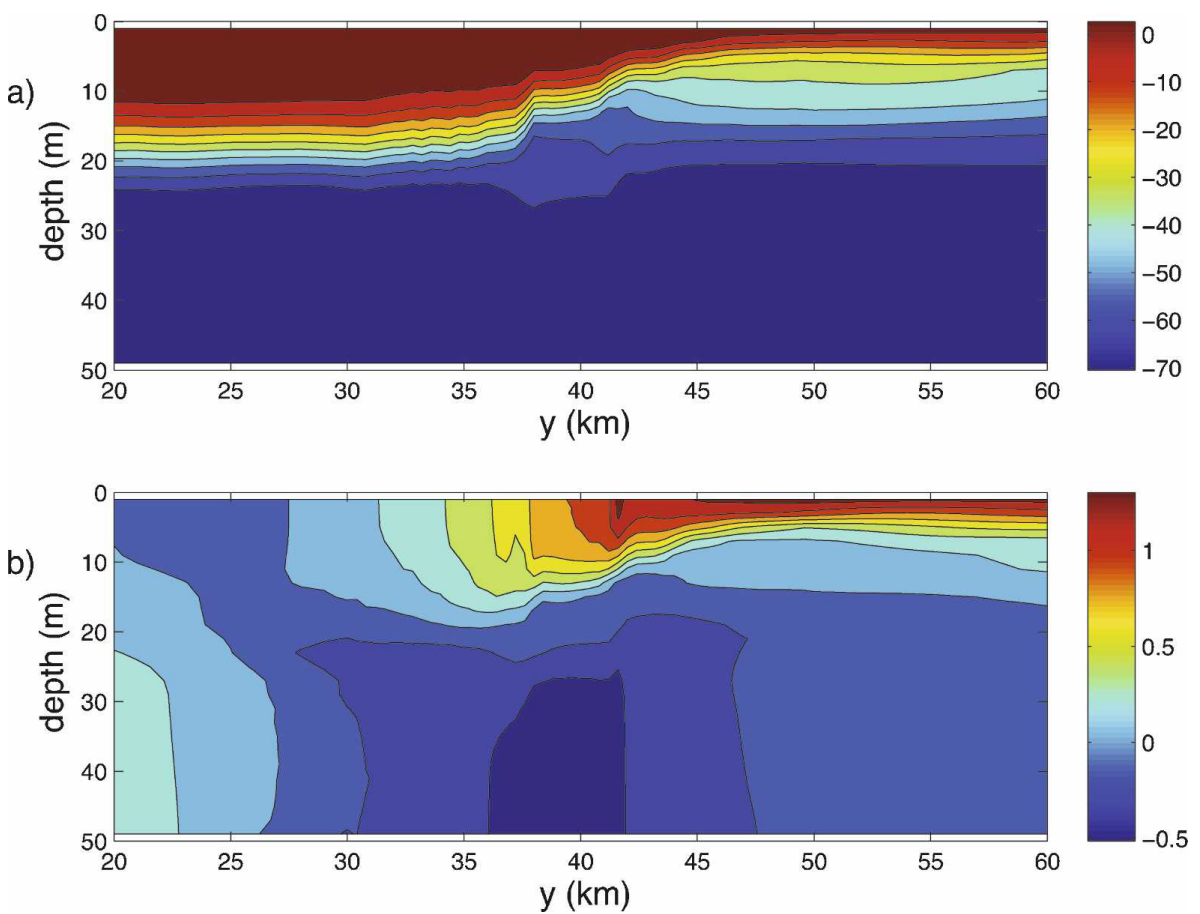

FIG. 11. Section of mean properties taken through the strait for $\left(\right.$ a) $T\left({ }^{\circ} \mathrm{C}\right)$ and (b) $v\left(\mathrm{~m} \mathrm{~s}^{-1}\right)$ for run $\mathrm{C} 1$.

strength of heat loss, the Coriolis parameter, and width of the sill have been varied. For the cases with a lateral constriction, the Coriolis parameter, strait width, and upper ocean thickness are varied. The time-average upper- and lower-layer temperatures and the thickness of the upper layer are calculated at the location where the composite Froude number is closest to unity. This is close to the shallowest or narrowest part of the strait in all cases. The differences between the inflowing and outflowing temperatures for each case are plotted in Fig. 14a as a function of the temperature scale defined by (25). The circles mark the flat-bottom cases, and the squares and diamonds mark the cases with a topographic sill. The prediction $\Delta T_{\mathrm{nh}}$ based on the theory that lacks hydraulic control (solid diagonal) reproduces the overall trend of increasing temperature anomaly with decreasing $H_{o}$, at least for the cases with a pure contraction, but the model consistently produces temperature anomalies that are much larger than this prediction. For the topographic sill cases, the theory underestimates the sensitivity to both changes in the Coriolis parameter and to the surface heat loss.

The temperature difference between the upper and lower layers, now nondimensionalized with the temperature scale (11), is shown in Fig. 14b as a function of the nondimensional upper-layer thickness $h_{1} / H$. The solid $\mathrm{U}$ curve indicates the temperature difference predicted by (10), which accounts for the hydraulic control and the marginal sea heat balance. As before, predicted maximal exchange states for various sill heights lie within the thickened portion, with zero sill height (a pure contraction) at the left end and a limiting tall sill at the right. There is generally close agreement between the model and the theory, with most of the model runs

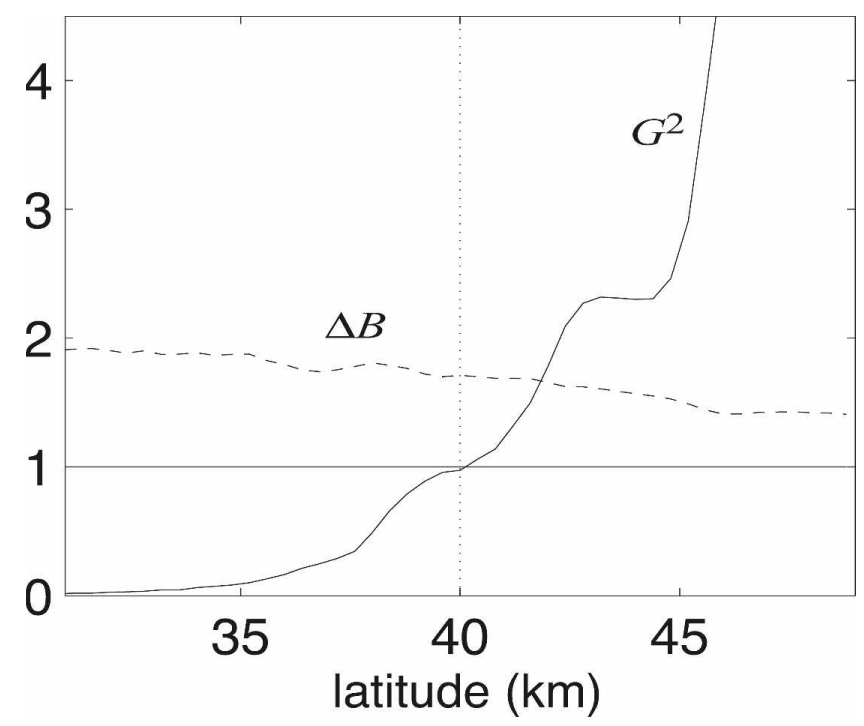

FIG. 12. Bernoulli function $\Delta B$ and the $G^{2}$ diagnosed from the model as a function of latitude from the open ocean $(0-30 \mathrm{~km})$ through the strait $(30-50 \mathrm{~km})$. Both quantities are measured along the right wall (facing into the strait). 

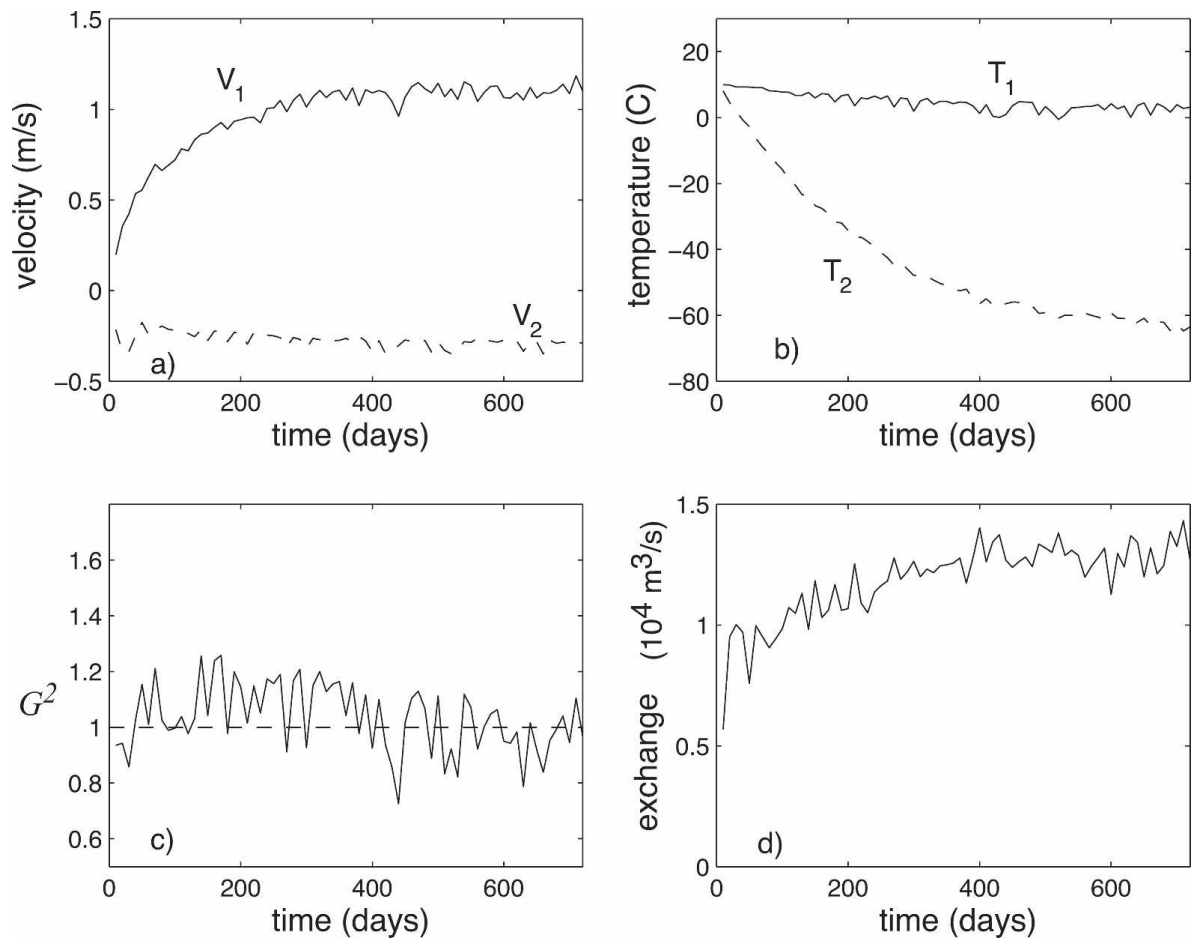

FIG. 13. Time series of (a) upper-layer (solid) and lower-layer (dashed) velocity, (b) upperlayer (solid) and lower-layer (dashed) temperature, (c) composite Froude number, and (d) exchange rate for run $\mathrm{C} 1$.

lying in the submaximal range. Three cases of maximal exchange, identified as having two distinct critical sections, were found, all with sills, and these are indicated by diamonds.

It is not difficult to show using (6), (9), and the critical condition $G^{2}=1$ that

$$
\tilde{Q}_{v}=\Delta \tilde{T}^{-1}
$$

where $\tilde{Q}_{v}=Q v \rho_{o}^{2 / 3} c_{P}^{1 / 3} /(g \alpha A Q)^{1 / 3} w^{2 / 3} H$ is a nondimensional measure of the volume exchange rate. This inverse relation is expected, because the heat exchange is proportional to the volume flux times the temperature difference. The $\tilde{Q}_{v}$ has been diagnosed from the model and plotted as asterisks on the same nondimensional scale as the temperature change in Fig. 14b. There is generally good agreement between the model values and those predicted by the theory (solid line). This provides another indication that the model runs are at a statistical equilibrium and that the flow is hydraulically controlled.

A more stringent test is to predict where along the $\mathrm{U}$ curve a particular model run will lie for a given value of the forcing parameter $\mu$. We start with cases with sills, for which numerical model data lie entirely along the right-hand branch of the $\mathrm{U}$ curve. The relationship between $h_{1} / H$ and $\mu$ is given graphically in Fig. 4 by the intersection of the appropriate dashed curve with the $\mathrm{U}$ curve. Analytically, the relation is obtained by eliminating $\Delta \tilde{T}$ between (10) and (16), and this results in the solid curve shown in Fig. 14c, the thickened portion of which corresponds to the range of maximal states for various sill heights. For the parameter range presented here, $\mu$ varies from 0.97 to 3.83 , the smaller values corresponding to stronger thermal forcing or more constricted exchange. The proportionality factor $\kappa$ in (5), which is the ratio of the width of the boundary current in the marginal sea to the internal deformation radius, has been set to 0.5 for all calculations. This is consistent with the modeled width in Fig. 6 and with the assumption that it is $O(1)$. For submaximal states (squares), the thickness of the upper layer compares reasonably well with that predicted by the theory.

According to the theory, maximal exchange conditions are reached when $\mu$ is decreased to a value of about $1.5 .^{2}$ At the transition, a second section of critical flow is predicted to occur near the opening of the strait into the marginal sea. If $\mu$ is decreased beyond the threshold value, this second critical section persists and

\footnotetext{
${ }^{2}$ The transitional value, which depends on the ratio of the sill height to the marginal sea depth, lies just below the transition between thin and thick curves in Fig. 12c.
} 

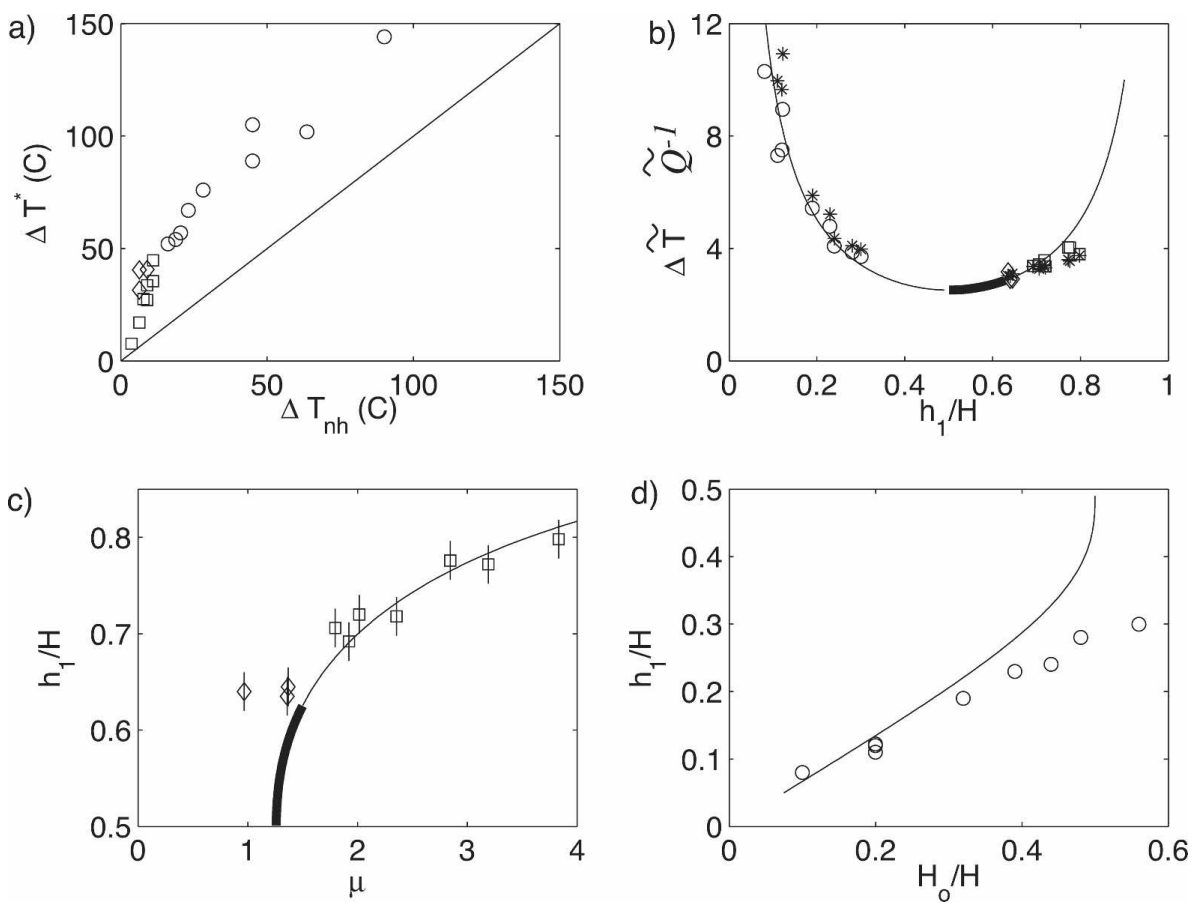

FIG. 14. Diagnostics from the series of model runs summarized in Table 1. Circles: constriction runs C1-C9; squares: sill runs S1-S7; diamonds: sill runs S8-S10. (a) $T_{1}-T_{2}$ for model runs compared to that predicted [Eq. (25)] in the absence of a hydraulic control. (b) $\Delta \tilde{T}$ for model runs compared to theory (10) leading to the U curve. We also show the nondimensional transport $\tilde{Q}$ [Eq. (26)] for model runs (asterisks) plotted against the theory $\left(\tilde{Q}=\Delta \tilde{T}^{-1}\right)$. The thick portion of the $U$ curve corresponds to the range of maximal flows for various sill depths. (c) Diagnosed upper-layer thickness as a function of the forcing parameter $\mu$ for calculations with a sill. The theory based on (10) and (16) is represented by the solid curve, the thickened portion of which corresponds to maximal flows. The vertical bars on each symbol indicate the thickness of one model level as a measure of the model uncertainty. (d) Upper-layer thickness as a function of the open ocean thickness $H_{o}$, with circles diagnosed from the model and the curve from the theory (22) for flow through a pure contraction with shallow inflow.

the flow into the marginal sea develops a hydraulic jump or some other dissipative form of transition. The upper-layer thickness $h_{1} / H$ and nondimensional temperature difference $\Delta \tilde{T}$ are predicted to remain at their values for maximal flow, and the dimensional volume exchange rate and temperature difference are expected to obey (18) and (19) and lie on the U curve. In Fig. 14c, these solutions should lie on a horizontal line to the left of the transition point. Numerical solutions with two critical sections are indicated by diamonds, and these do indeed lie to the left of, though slightly above, the maximal transition in Fig. 14c, along a line of nearly constant $h_{1} / H$. (Also, as shown in Fig. 14b, the corresponding values of $h_{1} / H$ and $\Delta \tilde{T}$ do remain stuck to the U curve.) The moderate departures from theoretical expectations may be a result of the unsteady nature of the exchange flow or of unanticipated sources of dissipation.

Previous laboratory studies of hydraulically controlled exchange flow between basins (e.g., Finnigan and Ivey 1999, 2000; Whitehead et al. 2003) tend to produce states that lie on just one branch of the $U$ curve. Our numerical runs with sills produced states on the right-hand branch, as expected from the theory. To produce left-hand branch solutions, we used a pure contraction and restore the open temperature over a limited upper-thickness $H_{o}$ of the total water depth $H$. The resulting states are indicated by circles and asterisks in Fig. 14b. The theory that relates the relative upper-layer thickness $h_{1} / H$ in the constriction to $H_{o} / H$ is given by (22), and the corresponding curve is plotted in Fig. 14d. Maximal exchange is approached at the right extent $\left(h_{1} / H \rightarrow 0.5\right)$ of the curve, where the slope becomes infinite. In principle, flow states should track this curve to the maximal point, then veer off along the horizontal line $h_{1} / H$. Our data track the curve reasonably well for $H_{o} / H<0.4$ but veer off before the theoretical maximal threshold $H_{o} / H=0.5$ is reached. This may be an indication that the flow is settling into a state of maximal exchange that is somehow influenced by 
friction, unsteadiness, or rotation and that arises short of the theoretical limit.

\section{Discussion}

We have presented an analytical model that predicts the volume exchange, the outflow temperature, and the layer depth structure for a marginal sea that is separated from the ocean by a highly constricted strait. Input parameters include the inflowing surface layer temperature, the heat loss over the marginal sea, the sill depth $H$, and an $O(1)$ dimensionless parameter $(\kappa)$, indicating the ratio of the marginal sea boundary layer width to the Rossby radius of deformation.

Although it is not a direct input into the model, the ratio of $H$ to the marginal sea depth $H_{\mathrm{ms}}$ is relevant. Its value determines whether a given solution is submaximal or maximal. Paleoceanographic applications may involve substantial changes in $H / H_{\mathrm{ms}}$ due to sea level variations, and these may imply transitions between submaximal and maximal states. In the limit of a very shallow sill $\left(H / H_{\mathrm{ms}} \rightarrow 0\right)$, often taken in idealized models, changes in $H$ cannot cause a transition from maximal to submaximal conditions, or vice versa. Thus, a reader who wishes to use our model in a paleoceanographic context should not operate in this limit.

The temperature differences found in our model simulations are considerably larger than those observed in Gibraltar or in the Bab al Mandab straits. However, if attention is restricted to cases with sills, and if the comparison is made in terms of density rather than temperature, the model results are not unrealistic. For example, Gibraltar has a salinity difference of about 4 psu, equivalent to about $17^{\circ} \mathrm{C}$ in terms of density change. This approximates the temperature difference of the sill flow shown in Fig. 7. On the other hand, density differences for cases with pure contractions are generally larger than observed. It may be significant that all of the major potential applications (the Mediterranean, Red, and Nordic seas, etc.) have significant sills. Calculations with smaller $\Delta T$ do not reach critical control.

To keep the discussion reasonably simple, we have chosen to restrict attention to one of two simple and distinct strait geometries: the pure sill and the pure width contraction. Solutions along the left branch of the $\mathrm{U}$ curve are possible only in the latter setting. A more realistic geometry would allow smooth, simultaneous variations in width and bottom depth, with the sill and narrows possibly occurring at different locations. This situation might relax restrictions concerning branches of the $U$ curve.

Although the agreement of the present model with our numerical simulations is generally good, it is less so in cases in which the flow of information is from the ocean into the marginal sea. Of our two permissible strait geometries, such states can occur only in the presence of a pure contraction and correspond to a relatively shallow upper layer. These states are dynamically consistent but less intuitive: the inflow of information from the open ocean runs counter to the idea that marginal seas are self-contained and largely immune from external processes. There is evidence that such states may occur over certain phases of the tides and/or periods of enhanced barotropic flow into the Strait of Gibraltar (Armi and Farmer 1988). Our theory for these solutions has good agreement with numerical simulations when the upper-layer thickness is quite small. However, as the upper layer is encouraged to become thicker (and the flow approaches the theoretical maximal exchange limit), the agreement worsens. Maximal conditions appear to be reached well before the theoretical threshold, and we have yet to find the multiple states predicted for the case of a pure contraction. Possible explanations include the effects of time dependence, nearly always present in the strait, or of dissipation that is not accounted for in the theory. Alternative methods of forcing the flow might lead to realization of the multiple states.

Further refinements of our model, which are possible, in principle, at some cost in terms of algebraic complexity, include incorporation of a salt budget and a provision for rotational effects within the strait. The latter could be accomplished by replacing the critical condition (1) by a two-layer generalization for rotating flow with zero potential vorticity:

$$
G_{r}^{2}=\frac{\bar{V}_{1}^{2} \bar{h}_{2}+\bar{V}_{2}^{2} \bar{h}_{1}-\frac{w^{2} f^{2}}{12 g^{\prime}}\left(\bar{V}_{2}-\bar{V}_{1}\right)^{2}}{g^{\prime}\left(\bar{h}_{1} \bar{h}_{2}-\frac{W^{2} f^{2}}{12 g^{\prime}} H\right)}=1
$$

(see Timmermans and Pratt 2005). Here, the overbars indicate cross-channel averages.

Acknowledgments. This work was supported by the National Science Foundation under Grants OCE-0525729 and OCE-0423975. We thank the two reviewers for their constructive comments.

\section{APPENDIX A}

\section{Case of a Small Basin}

Suppose that the boundary current encircles the basin, so that there is some recirculating flow around the rim. The upper-layer inflow from the strait no longer balances the boundary layer volume flux: in fact, the influx may be quite small in comparison. This case was 
considered by Spall (2004) and corresponds to his $\varepsilon<$ 1. Our model begins with his interior heat budget, in which the surface heat loss is balanced by an eddy heat flux from the boundary current:

$$
\begin{aligned}
\frac{A Q}{\rho_{o} c_{p}} & =C H_{b} \overline{u^{\prime} T^{\prime}}, \\
& =C H_{b} c V_{b}\left(T_{i n}-T_{o}\right),
\end{aligned}
$$

where $C$ is the marginal sea circumference. The second step results from a parameterization of baroclinic instability in the boundary current (Spall 2004) with diffusivity coefficient $c$. Combining the last results with the global heat budget (2) leads to

$$
V_{1} h_{1} W=c C_{b} V_{b} .
$$

This relation replaces (7), which is no longer valid. One may now work through the same steps outlined in section $2 \mathrm{~d}$, calculating expressions for $V_{1}, H_{b}$, and $V_{b}$ as functions of $T_{1}-T_{2}$ and $h_{1} / H$ and conserving wall energy between the most constricted section and the boundary current. The only difference is that (A2) is used in place of (7). The result is again (16), but with $\mu$ defined by

$$
\mu=\left(\frac{W \kappa}{c C}\right)^{2 / 3}\left(1+\frac{1}{2 \kappa^{2}}\right) .
$$

Therefore, all the curves and conclusions with regard to Fig. 4 continue to hold. Note that $\mu$ decreases as the basin size increases, or as $W$ decreases, which is consistent with the notion that larger or more strongly choked basins are more readily overmixed.

\section{APPENDIX B}

\section{Calculation of the Value of $h_{1} / H$ for Maximal Exchange with a Sill}

Let $H_{\mathrm{e}}$ denote the (constant) water depth in the marginal sea, also the depth at the entrance (denoted $e$ ) to the constant-width strait leading to the open ocean. Then, under conditions of maximal exchange, the twolayer flow is hydraulically critical at the entrance and at the sill. Using Eqs. $(5.3 .1,2)$ of Pratt and Whitehead (2007), conservation of volume flux and of the Bernoulli function between the entrance and sill sections lead to

$$
\frac{F_{1}^{-2 / 3}+\left(1-F_{1}^{2}\right)^{-1 / 3}}{F_{1 e}^{-2 / 3}+\left(1-F_{1 e}^{2}\right)^{-1 / 3}}=\frac{H}{H_{e}}
$$

and

$$
\begin{aligned}
\frac{1}{2} F_{1}^{4 / 3} & -\frac{1}{2}\left(1-F_{1}^{2}\right)^{2 / 3}+F_{1}^{-2 / 3}=\frac{1}{2} F_{1 e}^{4 / 3}-\frac{1}{2}\left(1-F_{1 e}^{2}\right)^{2 / 3} \\
+ & F_{1 e}^{-2 / 3},
\end{aligned}
$$

where $F_{1}$ and $F_{1 \mathrm{e}}$ are the upper-layer Froude numbers at the sill and entrance. Elimination of the latter between the above two equations gives, in principle, the value of $F_{1}$ in terms of the known $H / H_{e}$. The lowerlayer Froude number at the sill can be calculated from the critical condition as $F_{2}^{2}=1-F_{1}^{2}$, and the ratio of the upper-layer depth $h_{1}$ to the sill depth $H$ can then be found using the definitions of the Froude numbers and the constraint of zero net volume flux according to

$$
\frac{h_{1}}{H}=\left[\frac{F_{1}^{2 / 3}}{\left(1-F_{1}^{2}\right)^{1 / 3}}+1\right]^{-1} .
$$

We have already noted that $h_{1} / H=0.5$ for the case of a pure contraction $\left(H / H_{e} \rightarrow 1\right)$. In the opposite limit, that of a "tall" sill $\left(H / H_{e} \rightarrow 1\right)$, the lower layer at the entrance becomes inactive and the critical condition then implies that $F_{1 \mathrm{e}} \rightarrow 1$. Here, (B2) then gives $F_{1}=$ .420 and (B3) in turn gives $h_{1} / H=0.625$. This range $0.5<h_{1} / H<0.625$ corresponds to the thickened portion of the U curve (Figs. 4, 14.)

\section{REFERENCES}

Armi, L., 1986: The hydraulics of two flowing layers of different densities. J. Fluid Mech., 163, 27-58.

_- - and D. M. Farmer, 1986: Maximal two-layer exchange through a contraction with barotropic net flow. J. Fluid Mech., 164, 27-51.

— the Strait of Gibraltar. Prog. Oceanogr., 21, 1-105.

Baines, P. G., 1995 Topographic Effects in Stratified Flows. Cambridge University Press, 482 pp.

Brocard, D. N., and D. R. F. Harleman, 1980: Two-layer model for shallow horizontal connective circulation. J. Fluid Mech., 100, 129-146.

Bryden, H. L., and H. M. Stommel, 1984: Limiting processes that determine basic features of the circulation in the Mediterranean Sea. Oceanol. Acta, 7, 289-296.

Dalziel, S. B., 1991: Two-layer hydraulics: A functional approach. J. Fluid Mech., 223, 135-163.

Farmer, D. M., and L. Armi, 1986: Maximal two-layer exchange flow over a sill and through a combination of a sill and contraction with barotropic flow. J. Fluid Mech., 164, 53-76.

Finnigan, T. D., and G. N. Ivey, 1999: Submaximal exchange between a convectively forced basin and a large reservoir. $J$. Fluid Mech., 378, 357-378.

- and - 2000: Convectively driven exchange flow in a stratified sill-enclosed basin. J. Fluid Mech., 418, 313-338.

Grimm, T., and T. Maxworthy, 1999: Buoyancy-driven mean flow in a long channel with a hydraulically constrained exit condition. J. Fluid Mech., 398, 155-180.

Iovino, D., F. Straneo, and M. A. Spall, 2008: On the effect of a sill on dense water formation in a marginal sea. J. Mar. Res., in press.

Marshall, J., C. Hill, L. Perelman, and A. Adcroft, 1997: Hydrostatic, quasi-hydrostatic, and non-hydrostatic ocean modeling. J. Geophys. Res., 102, 5733-5752.

Pratt, L. J., and J. A. Whitehead, 2008: Rotating Hydraulics: Nonlinear Topographic Effects in the Ocean and Atmosphere. Springer, $582 \mathrm{pp}$. 
Price, J., and J. Yang, 1998: Marginal sea overflows for climate simulations. Ocean Modeling and Parameterization, E. P. Chassignet and J. Verron, Eds., NATO Sciences Series, Vol. 516, Kluwer Academic, 155-170.

Siddall, M., D. A. Smeed, S. Mattthiesen, and E. J. Rohling, 2002: Modeling the seasonal cycle of the exchange flow in Bab el Mandab (Red Sea). Deep-Sea Res. I, 49, 1551-1569.

,-- C. Hemleben, E. J. Rohling, I. Schmelzer, and W. R. Peltier, 2004: Understanding the Red Sea response to sea level. Earth Planet. Sci. Lett., 225, 421-434.

Smagorinsky, J., 1963: General circulation experiments with the primitive equations: I. The basic experiment. Mon. Wea. Rev., 91, 99-164.

Spall, M. A., 2003: On the thermohaline circulation in flat bottom marginal seas. J. Mar. Res., 61, 1-25.

- 2004: Boundary currents and water mass transformation in marginal seas. J. Phys. Oceanogr., 34, 1197-1213.
Stommel, H. M., and H. G. Farmer, 1953: Control of salinity in an estuary by a transition. J. Mar. Res., 12, 13-20.

Straneo, F., 2006: On the connection between dense water formation, overturning, and poleward heat transport in a convective basin. J. Phys. Oceanogr., 36, 1822-1840.

Timmermans, M.-L. E., and L. J. Pratt, 2005: Two-layer exchange flow between two deep basins: Theory and application to the Strait of Gibraltar. J. Phys. Oceanogr., 35, 1568-1592.

Walin, G., G. Broström, J. Nilsson, and O. Dahl, 2004: Baroclinic boundary currents with downstream decreasing buoyancy: A study of an idealized Nordic Seas system. J. Mar. Res., 62, 517-543.

Whitehead, J. A., M. L. E. Timmermans, W. G. Lawson, S. N. Bulgakov, A. M. Zatarian, J. F. A. Medina, and J. Salzig, 2003: Laboratory studies of thermally and/or salinity driven flows with partial mixing 1 . Stommel transitions and multiple flow states. J. Geophys. Res., 108, 3036, doi:10.1029/ 2001JC000902. 